OPEN ACCESS

Edited by:

Julien Licchesi,

University of Bath, United Kingdom

Reviewed by:

Christopher West,

University of Georgia, United States

Francisco Velazquez,

University of Buenos Aires, Argentina

*Correspondence:

Robert J. Huber roberthuber@trentu.ca

tThese authors have contributed equally to this work

Specialty section:

This article was submitted to Integrative Physiology, a section of the journal Frontiers in Physiology

Received: 02 December 2021 Accepted: 03 February 2022 Published: 01 March 2022

Citation: Kim WD, Mathavarajah S and Huber RJ (2022) The Cellular and Developmental Roles of Cullins, Neddylation, and the COP9

Signalosome in Dictyostelium discoideum.

Front. Physiol. 13:827435. doi: 10.3389/fphys.2022.827435

\section{The Cellular and Developmental Roles of Cullins, Neddylation, and the COP9 Signalosome in Dictyostelium discoideum}

\author{
William D. Kim ${ }^{1 \dagger}$, Sabateeshan Mathavarajah ${ }^{2 \dagger}$ and Robert J. Huber ${ }^{3 * \dagger}$ \\ ${ }^{1}$ Environmental and Life Sciences Graduate Program, Trent University, Peterborough, ON, Canada, ${ }^{2}$ Department of \\ Pathology, Dalhousie University, Halifax, NS, Canada, ${ }^{3}$ Department of Biology, Trent University, Peterborough, ON, Canada
}

Cullins (CULs) are a core component of cullin-RING E3 ubiquitin ligases (CRLs), which regulate the degradation, function, and subcellular trafficking of proteins. CULs are posttranslationally regulated through neddylation, a process that conjugates the ubiquitin-like modifier protein neural precursor cell expressed developmentally downregulated protein 8 (NEDD8) to target cullins, as well as non-cullin proteins. Counteracting neddylation is the deneddylase, COP9 signalosome (CSN), which removes NEDD8 from target proteins. Recent comparative genomics studies revealed that CRLs and the CSN are highly conserved in Amoebozoa. A well-studied representative of Amoebozoa, the social amoeba Dictyostelium discoideum, has been used for close to 100 years as a model organism for studying conserved cellular and developmental processes owing to its unique life cycle comprised of unicellular and multicellular phases. The organism is also recognized as an exceptional model system for studying cellular processes impacted by human diseases, including but not limited to, cancer and neurodegeneration. Recent work shows that the neddylation inhibitor, MLN4924 (Pevonedistat), inhibits growth and multicellular development in $D$. discoideum, which supports previous work that revealed the cullin interactome in $D$. discoideum and the roles of cullins and the CSN in regulating cellular and developmental processes during the $D$. discoideum life cycle. Here, we review the roles of cullins, neddylation, and the CSN in $D$. discoideum to guide future work on using this biomedical model system to further explore the evolutionarily conserved functions of cullins and neddylation.

Keywords: COP9 signalosome, cullins, Dictyostelium discoideum, neddylation, SCF complex

\section{NEDDYLATION, CULLINS, AND THE COP9 SIGNALOSOME}

The continual turnover of proteins through degradation maintains cell homeostasis, facilitates signal transduction, and allows for progression through the cell cycle. One of the pathways cells use to degrade proteins involves the proteasome, where ubiquitin is the tag that marks proteins for degradation. In addition to ubiquitin, there are also ubiquitin-like modifiers that target both proteins and lipids to control their subcellular localization, macromolecular interactions, 
and enzymatic activity (Cappadocia and Lima, 2018). Known ubiquitin-like modifiers include small ubiquitin-like modifier (SUMO), ubiquitin fold modifier 1 (UFM1), ubiquitin-related modifier 1 (URM1), ubiquitin-like modifier HUB1, and neural precursor cell expressed developmentally downregulated protein 8 (NEDD8; Vierstra, 2012). NEDD8 is highly conserved across eukaryotes (Figure 1) and is conjugated to target proteins at a near-terminal lysine residue (N-term or $\mathrm{C}$-term) through a process known as neddylation. The modification was first observed in the Saccharomyces cerevisiae S phase kinase-associated protein 1 (Skp1)-Cullin-F-box (SCF) complex, where Rub1, the $S$. cerevisiae ortholog of human NEDD8, was found conjugated to Cdc53p, the S. cerevisiae ortholog of cullin 1 (CUL1; Lammer et al., 1998; Liakopoulos et al., 1998). In the SCF complex,

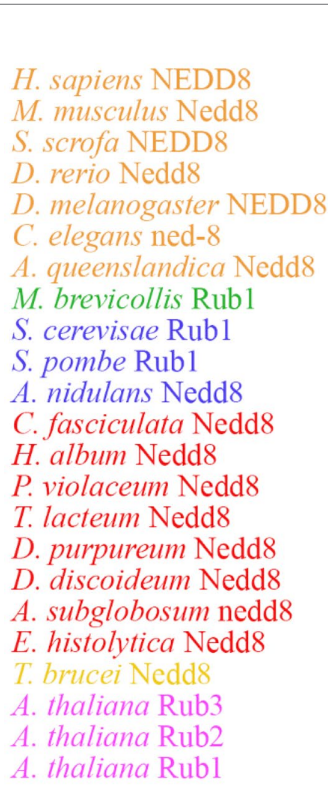

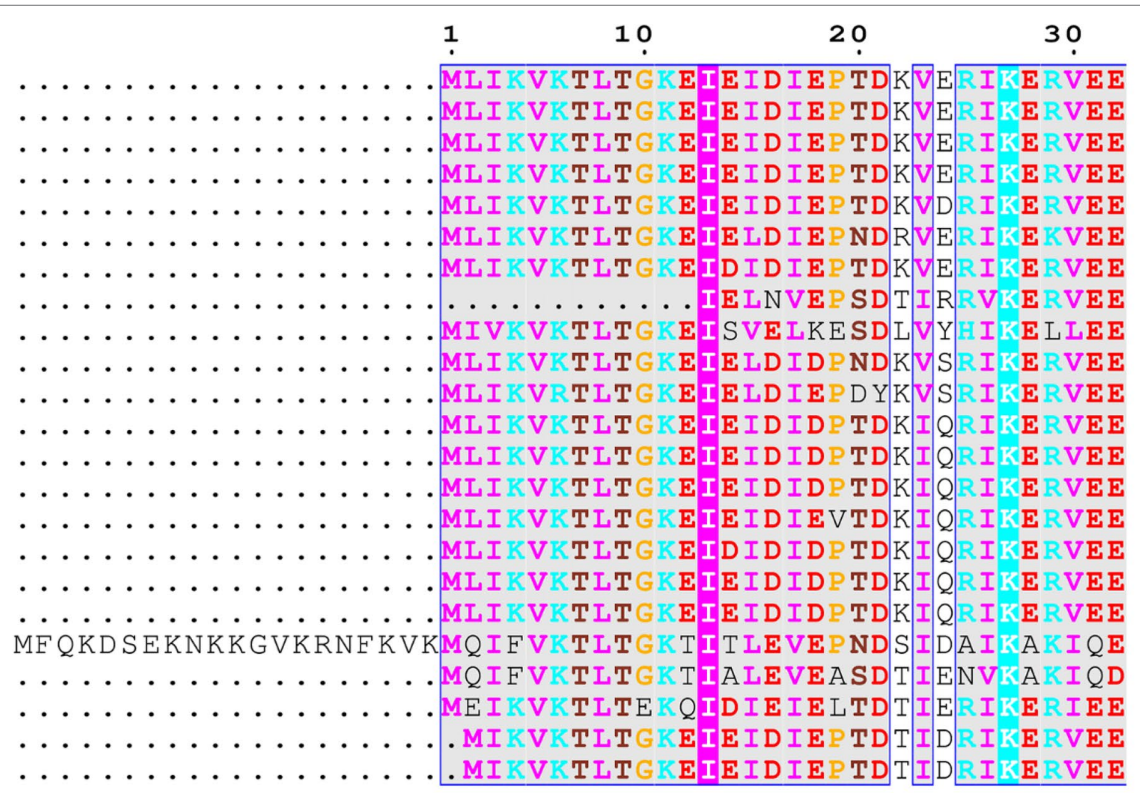

H. sapiens NEDD8
M. musculus Nedd8
S. scrofa NEDD8
D. rerio Nedd8
D. melanogaster NEDD8
C. elegans ned-8
A. queenslandica Nedd8
M. brevicollis Rub1
S. cerevisae Rub1
S. pombe Rub1
A. nidulans Nedd8
C. fasciculata Nedd8
H. album Nedd8
P. violaceum Nedd8
T. lacteum Nedd8
D. purpureum Nedd8
D. discoideum Nedd8
A. subglobosum nedd8
E. histolytica Nedd8
T. bricei Nedd8
A. thaliana Rub3
A. thaliana Rub2
A. thaliana Rub1

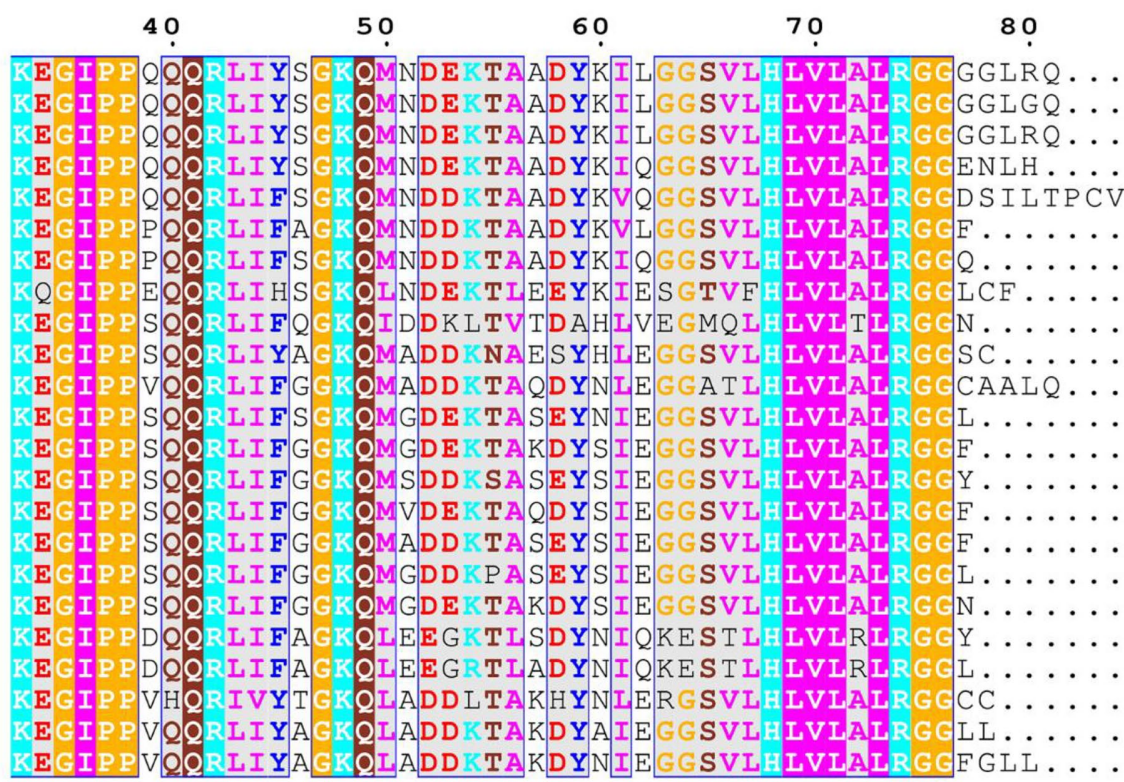

$\Delta \mathbf{\Lambda}$

FIGURE 1 | Neural precursor cell expressed developmentally downregulated protein 8 (NEDD8) is conserved across eukaryotes. Alignment of NEDD8 ortholog protein sequences in different eukaryote species, including those in plants (pink), amoebozoa (red), fungi (blue), and animals (orange). For plants, the upstream ubiquitin sequence was trimmed from the NEDD8 orthologs. Sequences were aligned on MEGA7 using the MUSCLE alignment and displayed using ESPript 3.0. Colored residues indicate physiochemical properties and conservation at a position. The C-terminal "RGG" proteolytic processing motif is marked by red arrowheads and the NEDD8 overhang sequence that is cleaved is indicated by the blue line. 
the linker protein SKP1 bridges the interaction between the cullin and F-box protein, which targets specific substrates for ubiquitination (Zimmerman et al., 2010). Cullins then serve as the scaffold for multi-subunit ubiquitin ligases. SCF is a member of the cullin-RING E3 ubiquitin ligase (CRL) superfamily, which plays important roles in regulating a variety of proteins including transcription factors, cell cycle regulators, DNA damage response/repair proteins, and growth factor receptors (Schwechheimer and Villalobos, 2004; Vodermaier, 2004; Willems et al., 2004; Ardley and Robinson, 2005; Bosu and Kipreos, 2008; de Bie and Ciechanover, 2011; Li and Jin, 2012; Chung and Dellaire, 2015; Qi and Ronai, 2015; Gilberto and Peter, 2017; Serrano et al., 2018). In addition, neddylation plays an important role in regulating the activity of subunits that form the proteasome and ribosomes (Xirodimas et al., 2008). During proteotoxic stress, neddylation promotes ribosomal protein accumulation in the nucleus to protect the proteasome system and prevent dysfunction (Maghames et al., 2018). During oxidative stress, neddylation regulates poly(ADP-ribose) polymerase 1 (PARP1) activity to delay the initiation of PARP1dependent cell death (Keuss et al., 2019). Thus, neddylation has multiple essential functions in the cell.

Many aspects of neddylation mirror and incorporate pathways that are associated with ubiquitination. Akin to ubiquitin, once NEDD8 is translated into an inactive precursor form, it requires cleavage of its short C-terminal amino acid extension (five amino acids in humans) to generate the mature form of the protein (Figures 1, 2; Kamitani et al., 1997). Mature NEDD8 has an accessible C-terminal glycine residue positioned at amino acid 76 that is used to conjugate NEDD8 to the lysine of a target protein (Figure 1; Kamitani et al., 1997). NEDD8 $\mathrm{C}$-terminal cleavage occurs through the actions of ubiquitin C-terminal hydrolase isozyme (UCH) L1 (UCHL1) and UCHL3 (both belonging to the C12 family of peptidases; Figure 2). UCHs also cleave the C-terminal extensions of ubiquitin (Wada et al., 1998; Johnston et al., 1999; Linghu et al., 2002; Hemelaar et al., 2004; Frickel et al., 2007; Yu et al., 2011). While UCHL1 and UCHL3 both bind to NEDD8, the hydrolytic processing of NEDD8 is carried out by UCHL3 (Wada et al., 1998). Sentrin-specific protease 8 (SENP8/DEN1; belonging to the C48 family of peptidases) is another NEDD8 processing enzyme that exclusively targets NEDD8 and not ubiquitin (Figure 2; Gan-Erdene et al., 2003; Mendoza et al., 2003; Wu et al., 2003; Shen et al., 2005; Chan et al., 2008; Shin et al., 2011). Like UCHL3, SENP8 also cleaves NEDD8 at the 5-amino acid C-terminal extension suggesting the functions of the two enzymes are redundant (Mendoza et al., 2003).

Like ubiquitination, the conjugation of mature NEDD8 to target proteins follows an E1-E2-E3 cascade (Figure 2; Kawakami et al., 2001; Pan et al., 2004; Huang et al., 2005; Saha and Deshaies, 2008). After NEDD8 is cleaved, it is adenylated and activated by $\mathrm{E} 1$ in an adenosine triphosphate (ATP)-dependent mechanism (Bohnsack and Haas, 2003; Walden et al., 2003). E1 is a heterodimer of NEDD8-activating enzyme E1 regulatory subunit (NAE1) and ubiquitin-activating enzyme 3 (UBA3; Gong and Yeh, 1999; Bohnsack and Haas, 2003; Walden et al., 2003). Within E1, NEDD8 resides between NAE1 and UBA3 but binds directly with the latter. E1 then transfers NEDD8 to $\mathrm{E} 2$, which is comprised of ubiquitin-conjugating enzyme (UBE) 2F (UBE2F) and UBE2M (Osaka et al., 1998; Gong and Yeh, 1999; Huang et al., 2009). Both UBE2F and UBE2M can bind NEDD8 (Gong and Yeh, 1999; Huang et al., 2005, 2009). E2 receives assistance in the final step of neddylation from a few E3 ligases such as ring box 1/regulator of cullins 1 (RBX1/ROC1), ring box 2/regulator of cullins 2 (RBX2/ ROC2), and defective in cullin neddylation 1 (DCUN1D1), to transfer NEDD8 to the lysine of target proteins (e.g., cullins) and initiate CRL assembly (Kamura et al., 1999; Petroski and Deshaies, 2005; Duda et al., 2008; Saha and Deshaies, 2008; Huang et al., 2009; Scott et al., 2010). Previous work showed that RBX1 and DCUN1D1 interact with the NEDD8-UBE2M intermediate to neddylate CUL1, CUL3, CUL3, and CUL4 (Kim et al., 2008; Huang et al., 2009). CUL5 neddylation is carried out by RBX2 in association with UBE2F, but unlike RBX1, it is not known if DCUN1D1 also participates in the conjugation (Huang et al., 2009). Finally, CUL7 and CUL9 have been shown to bind to RBX1 (Andrews et al., 2006). Like ubiquitination, proteins can be polyneddylated (Jones et al., 2008). However, unlike ubiquitin, which has over 10,000 targets in humans, NEDD8 appears to be conjugated to a shorter list of proteins. For example, Jones et al. (2008) performed a targeted proteomics analysis to identify 496 NEDD8-modified and associated proteins in HEK293 cells, which included all human cullins. In addition, a recent study identified 1,101 unique neddylation sites on 620 human proteins in HEK293 cells (Lobato-Gil et al., 2021). Cullins undergo neddylation at a consensus C-terminal neddylation motif [(IL)(VIT)(RQ)(IS) $(\mathrm{MLV}) \mathrm{K}(\mathrm{MAS})(\mathrm{RHE})]$ and are conjugated specifically to a lysine residue found within the motif (Mikus and Zundel, 2005). Therefore, neddylation represents a unique but smaller pool of ubiquitin-like modification within the eukaryotic cell, where cullins are the major targets.

The dynamic assembly of CRL complexes is modulated by cullin-associated NEDD8-dissociated protein 1 (CAND1), which functions as a SKP1/F-box protein exchange factor for CUL1 (as well as other cullins; Figure 2; Zheng et al., 2002; Dubiel et al., 2013; Pierce et al., 2013; Liu et al., 2018). Neddylation of CUL1 and subsequent binding of SKP1 and a F-box protein causes the displacement of CAND1, thus allowing for CRL assembly (Liu et al., 2002; Zheng et al., 2002). Without the incorporation of CAND1, there is inefficient degradation of target proteins. Neddylation also contributes to the enzymatic activity of the SCF complex by causing a conformational shift to improve ubiquitin transfer activity and E2 recruitment (Saha and Deshaies, 2008). The important role of this process is highlighted in studies that showed that the complete loss of neddylation is lethal (with S. cerevisiae being the exception; Lammer et al., 1998; Osaka et al., 2000; Tateishi et al., 2001). In humans, abnormalities in neddylation are linked to a variety of pathological conditions including cancer, neurodegeneration, autoimmune diseases, and other inflammatory diseases (Chen et al., 2012; Enchev et al., 2015; Ehrentraut et al., 2016; Ying et al., 2018). Together, these findings suggest that neddylation is a key component of CRL regulation, and when aberrant, 


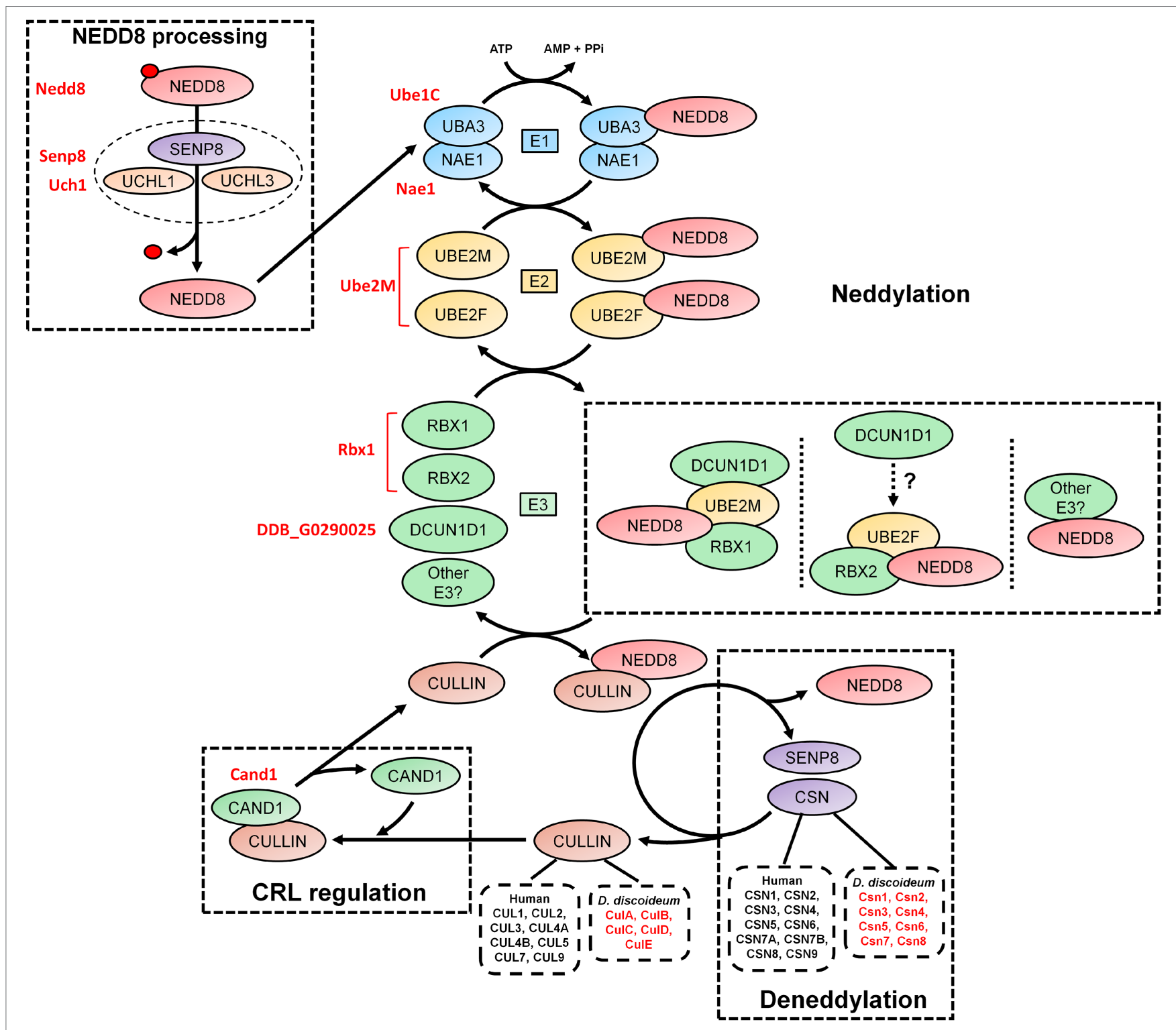

FIGURE 2 | An overview of the neddylation and deneddylation pathway. NEDD8 is processed into its matured form through both ubiquitin C-terminal hydrolase isozyme (UCH) and Sentrin-specific protease 8 (SENP8) proteolytic cleavage. NEDD8 is passed through a "baton" mechanism, where NEDD8 is adenylated and activated in an adenosine triphosphate (ATP)-dependent mechanism by E1, which is a heterodimer of NEDD8-activating enzyme E1 regulatory subunit (NAE1) and ubiquitin-activating enzyme 3 (UBA3). NEDD8 binds specifically to UBA3 within E1. UBA3 binds both ubiquitin-conjugating enzyme (UBE) 2F (UBE2F) and ubiquitinconjugating enzyme E2M (UBE2M) in E2 and transfers NEDD8 to both proteins. NEDD8 is finally attached to a substrate (i.e., cullins and non-neddylated proteins) by E3, which includes ring box 1 (RBX1), ring box 2 (RBX2), and other potential E3 ligases. In E3, NEDD8 loaded into UBE2M associates with RBX1 and defective in cullin neddylation 1 (DCUN1D1). NEDD8 loaded into UBE2F associates with RBX2, but the involvement of DCUN1D1 is unknown. Substrate neddylation displaces cullin-associated NEDD8-dissociated protein 1 (CAND1), which serves to regulate cullin-RING E3 ubiquitin ligases (CRL) assembly. NEDD8 is removed from the substrate via deneddylation, which involves the COP9 signalosome (CSN) complex and SENP8/DEN1. The Dictyostelium discoideum orthologs are displayed as red text beside the respective human protein. AMP, adenosine monophosphate; ATP, adenosine triphosphate; CAND1, cullin-associated NEDD8-dissociated protein 1; CSN, COP9 signalosome; CUL, cullin; E1, E1-NAE1 complex; E2, E2-conjugation complex; E3/CRL, E3-cullin-RING complex; NAE1, NEDD8 activating enzyme E1 subunit 1; NEDD8, neural precursor cell expressed developmentally downregulated protein 8; PPi, pyrophosphate; RBX, ring box; SENP8, sentrin-specific protease 8; UBA3, ubiquitin-activating enzyme 3; UBE, ubiquitin-conjugating enzyme; UCH, ubiquitin C-terminal hydrolase; and UCHL, ubiquitin C-terminal hydrolase isozyme.

contributes to the pathogenesis associated with many human diseases.

Deneddylation (removal of NEDD8 from proteins) occurs through the actions of the COP9 signalosome (CSN), which is composed of nine subunits in humans (CSN1-6, CSN7A/7B, and CSN8-9; Figure 2; Rao et al., 2020). SENP8/DEN1, which participates in NEDD8 processing (discussed above), also plays a role in disassembling CRLs (Wu et al., 2003; 
Shin et al., 2011). The CSN exists as two variant complexes containing CSN1-6, CSN8-9, and one of CSN7A or CSN7B, which have overlapping functions in the deneddylation of CRLs (Wang et al., 2021). However, CSN7B has been reported to have a unique function in adipogenesis and the DNA damage response (Huang et al., 2016; Wang et al., 2021). The CSN is conserved across eukaryotes including plants (e.g., Arabidopsis thaliana), invertebrates (e.g., Drosophila melanogaster and Caenorhabditis elegans), yeast (e.g., S. cerevisiae and Schizosaccharomyces pombe), fungi (e.g., Neurospora crassa and Aspergillus nidulans), and humans (Schwechheimer et al., 2001; Busch et al., 2003; Doronkin et al., 2003; Pintard et al., 2003; He et al., 2005; Wee et al., 2005; Wu et al., 2005; Cope and Deshaies, 2006; Schmidt et al., 2010). CSN complexes that contain fewer than nine subunits have been observed in different eukaryotic clades, suggesting that the protein architecture of the complex has been prone to changes over time (Braus et al., 2010). However, a consistent hallmark is the conservation of the CSN5 subunit among different eukaryotes.

As discussed above, the CSN subunits exhibit widespread abundance throughout both unicellular and multicellular eukaryotes (Barth et al., 2016). Amoebozoan genomes encode either a CSN with all the known subunits or all but CSN8 and CSN9 subunits (Barth et al., 2016). A representative model organism from Amoebozoa is the social amoeba, Dictyostelium discoideum, which was identified as a species with a genome encoding an intact eight subunit CSN (Rosel and Kimmel, 2006; Heidel et al., 2011). D. discoideum emerged at least 600 million years ago (an amorphea that diverged prior to the fungi-animal split) and has been studied for close to a century (Mathavarajah et al., 2017). Various cullins have been identified as key regulators of multicellular development in $D$. discoideum (Mohanty et al., 2001; Wang and Kuspa, 2002; Sheikh et al., 2015). Furthermore, components of the neddylation pathway in metazoans are conserved in D. discoideum (Figure 2; Table 1), and there is evidence supporting cullin neddylation during the life cycle (Sheikh et al., 2015). Here, we review the known and predicted roles of cullins and neddylation in $D$. discoideum to set the stage for future work that further examines how cullins and neddylation regulate conserved cellular and developmental processes.

\section{THE LIFE CYCLE OF DICTYOSTELIUM DISCOIDEUM}

D. discoideum belongs to a clade within the Amoebozoan known as the social amoebae, a term coined by Bonner (1949) after observing that unicellular $D$. discoideum amoebae could develop into multicellular fruiting bodies when prompted by starvation. In the 24-h asexual life cycle of D. discoideum, a starved population of amoebae aggregate to form complex multicellular structures in a time-dependent manner (Fey et al., 2007; Gaudet et al., 2008; Mathavarajah et al., 2017; Figure 3A). After aggregating to form a mound, cells rise above the surface to form a finger, which then falls on the surface to form a motile pseudoplasmodium, or slug (Raper, 1940; Brenner, 1977). As multicellular development continues, the slug forms a culminant where cells undergo terminal differentiation to form a fruiting body, the final stage of development (Marée and Hogeweg, 2001). The fruiting body is composed of a mass of differentiated spores that sit atop a slender stalk of differentiated stalk cells. During differentiation, $\sim 80 \%$ of the cells within the slug become pre-spore cells, which eventually differentiate into spores (Forman and Garrod, 1977). The other $\sim 20 \%$ differentiate into pre-stalk cells and become non-reproductive cells that comprise the stalk and two other segments of the fruiting body. These two other segments are composed of differentiated cells derived from the same cell type as the stalk cells and are referred to as the cup and basal disk cells (they constitute both the cup and basal structures of the fruiting body, respectively; Chen and Schaap, 2016). The D. discoideum life cycle highlights the evolution of many processes required for multicellular development, including but not limited to, cellcell communication, cell-cell adhesion, and differentiation.

\section{NEDDYLATION, CULLINS, AND THE CSN IN DICTYOSTELIUM DISCOIDEUM}

\section{Neddylation and Cullins in Dictyostelium discoideum}

In $D$. discoideum, nedd8 is expressed throughout development but peaks in expression after $4 \mathrm{~h}$ of development (Figure 3B). This expression profile overlaps with the expression profiles of most cullin genes in $D$. discoideum, which peak in expression during aggregation (Figure 3B). Unlike later diverging amorphea, the C-terminal extension is only one amino acid long for $D$. discoideum Nedd8 (Figure 1; Huber et al., 2021). The D. discoideum genome also encodes a protein homologous to UCHL1 and UCHL3 in humans, Uch1 (encoded by uch1), and an ortholog of human SENP8, Senp8 (encoded by senp8; Figure 2; Table 1; Huber et al., 2021).

The $D$. discoideum E1 complex is a heterodimer comprised of orthologs of mammalian NAE1 and UBA3 (Nae1 and Ube1C, respectively; Figure 2; Table 1). There is only one potential E2 encoded by ube $2 M$ (protein: Ube $2 \mathrm{M}$ ), which is similar in sequence to both UBE2M and UBE2F. The $D$. discoideum genome also encodes an ortholog of RBX1 and RBX2, Rbx1, that is proposed to function as an E3 in the organism, as well as an ortholog of DCUN1D1 (uncharacterized protein DDB0305617; Forman and Garrod, 1977). Finally, D. discoideum contains an ortholog of human CAND1 (Cand1). Together, the predicted neddylation pathway in $D$. discoideum shares similarities to the well-established pathway in metazoans.

In mammals, there are eight members of the cullin family (CUl1, CUl2, CUl3, CUl4A, CUl4B, CUl5, CUl7, and CUL9; Table 2; Sarikas et al., 2011). The D. discoideum genome encodes five proteins (CulA, CulB, CulC, CulD, and CulE encoded by $c u l A, c u l B, c u l C, c u l D$, and $c u l E$, respectively) that all share sequence similarity with human 
TABLE 1 | Sequence similarity between neddylation pathway proteins and CSN subunits in humans and Dictyostelium discoideum.

\begin{tabular}{|c|c|c|c|c|c|c|c|}
\hline Human protein (Uniprot ID) & Size (aa) & $\begin{array}{l}\text { Dictyostelium discoideum } \\
\text { ortholog (dictyBase gene ID) }\end{array}$ & Size (aa) & $\begin{array}{c}\text { Region of } \\
\text { similarity (aa) }\end{array}$ & $\begin{array}{l}\text { Identities } \\
(\%)^{*}\end{array}$ & $\begin{array}{l}\text { Positives } \\
\text { (\%)** }\end{array}$ & E-value \\
\hline \multicolumn{8}{|l|}{ NEDD8 processing } \\
\hline NEDD8 (Q15843) & 81 & Nedd8 (DDB_G0278711) & 77 & 76 & 82 & 92 & $9 E-26$ \\
\hline SENP8/DEN1 (Q96LD8) & 212 & Senp8 (DDB_G0278795) & 243 & 241 & 32 & 47 & $4 \mathrm{E}-26$ \\
\hline UCHL1 (P09936) & 223 & Uch1 (DDB_G0282007) & 255 & 221 & 47 & 66 & $3 E-54$ \\
\hline UCHL3 (P15374) & 230 & & & 224 & 52 & 70 & $1 \mathrm{E}-65$ \\
\hline \multicolumn{8}{|l|}{ NAE1 heterodimer } \\
\hline NAE1/APPBP1 (Q13564) & 534 & Nae1 (DDB_G0287965) & 520 & 524 & 41 & 64 & $1 E-123$ \\
\hline UBA3 (Q8TBC4) & 463 & Ube1c (DDB_G0283891) & 442 & 441 & 49 & 68 & $1 E-128$ \\
\hline \multicolumn{8}{|l|}{ E2 } \\
\hline UBE2M/UBC12 (P61081) & 183 & Ube2M (DDB_G0281725) & 230 & 178 & 53 & 73 & $1 E-54$ \\
\hline UBE2F (Q969M7) & 185 & & & 175 & 33 & 53 & $6 \mathrm{E}-24$ \\
\hline \multicolumn{8}{|l|}{ E3 } \\
\hline RBX1/ROC1 (P62877) & 108 & Rbx1 (DDB_G0287629) & 104 & 97 & 86 & 92 & $2 \mathrm{E}-42$ \\
\hline RBX2/ROC2 (Q9UBF6) & 113 & & & 104 & 46 & 60 & $4 \mathrm{E}-20$ \\
\hline DCUN1D1 (Q96GG9) & 259 & Unnamed (DDB_G0290025) & 249 & 253 & 37 & 56 & $6 E-43$ \\
\hline \multicolumn{8}{|l|}{ Regulator of CRL assembly } \\
\hline CAND1 (Q86VP6) & 1,230 & Cand1 (DDB_G0274167) & 1,238 & 1,256 & 40 & 61 & 0 \\
\hline \multicolumn{8}{|l|}{ CSN subunits } \\
\hline CSN1/COPS1/GPS1 (Q13098) & 491 & Csn1 (DDB_G0283587) & 458 & 436 & 45 & 63 & $1 E-102$ \\
\hline CSN2/COPS2/TRIP15 (P61201) & 443 & Csn2 (DDB_G0289361) & 449 & 417 & 63 & 79 & $1 E-143$ \\
\hline CSN3/COPS3 (Q9UNS2) & 423 & Csn3 (DDB_G0291848) & 418 & 379 & 40 & 61 & $2 \mathrm{E}-76$ \\
\hline CSN4/COPS4 (Q9BT78) & 406 & Csn4 (DDB_G0293844) & 393 & 387 & 47 & 71 & 9E-95 \\
\hline CSN5/COPS5/JAB1 (Q92905) & 334 & Csn5 (DDB_G0284597) & 332 & 332 & 66 & 81 & $1 \mathrm{E}-130$ \\
\hline CSN6/COPS6/HVIP (Q7L5N1) & 327 & Csn6 (DDB_G0293180) & 309 & 288 & 41 & 64 & $2 \mathrm{E}-61$ \\
\hline CSN7A/COPS7A/DERP10 (Q9UBW8) & 275 & Csn7 (DDB_G0271282) & 259 & 194 & 41 & 68 & $1 \mathrm{E}-43$ \\
\hline CSN7B/COPS7B (Q9H9Q2) & 264 & & & 182 & 46 & 69 & $1 \mathrm{E}-41$ \\
\hline CSN8/COPS8 (Q99627) & 209 & Csn8 (DDB_G0275471) & 196 & 200 & 30 & 54 & $1 \mathrm{E}-24$ \\
\hline CSN9/COPS9 (Q8WXC6) & 57 & Could not be identified & - & - & - & - & - \\
\hline
\end{tabular}

BLASTp searches were performed using dictyBase. Human proteins were used as query sequences (E-value, 0.1; Matrix, BLOSUM62; Filter, no).

* Exact amino acid match.

**Similar amino acid match (e.g., both polar).

cullins (Table 2). Moreover, Sheikh et al. (2015) compared characteristic sequence motifs in cullins to show that CUL1 is most similar to CulA, CulE, and CulB, CUL3 is most similar to CulC, and CUL4B is most similar to CulD. BLASTp searches also show that the $D$. discoideum ortholog of anaphase promoting complex subunit 2 (Anapc2) shares limited sequence similarity with human CUL1, CUL2, CUL3, CUL4A, and CUL4B (Table 2). In mammals, anaphase promoting complex functions as an E3 ubiquitin ligase that regulates cell cycle progression by mediating ubiquitination and subsequent degradation of target proteins (Tang et al., 2001; Jin et al., 2008). Since only CulE has previously been validated as a neddylated protein, we examined the sequences of other $D$. discoideum cullins to determine whether the neddylation motif is conserved (Robert and Gouet, 2014; Kumar et al., 2016). The alignment revealed that the cullin neddylation motif is highly conserved between cullins from $D$. discoideum and humans (Figure 4). While the residues upstream of the lysine are identical and conserved in D. discoideum cullins, there are differences in the two downstream residues. Adjacent to the lysine ( +1 position), CulD has a threonine rather than adhering to the (MAS) amino acid sequence (methionine, alanine, or serine at the +1 position), indicating functional flexibility between the threonine and serine groups in $D$. discoideum. Similarly, CulE differs at the +2 position with a lysine residue that normally contains either an arginine, histidine, or glutamic acid residue [i.e., (RHE)]. Since there is a highly conserved neddylation motif present in each of CulA, CulB, CulC, CulD, and CulE, there is potential for Nedd8 to be conjugated to all $D$. discoideum cullins. Finally, the $D$. discoideum genome encodes orthologs of eight human CSN subunits (Table 1).

\section{Cullin Gene Expression During the Dictyostelium discoideum Life Cycle}

The $D$. discoideum cullin genes are differentially expressed during development and peak in expression at different times during the developmental program (Figure 3B). The expression of $c u l B, c u l C, c u l D$, and $c u l E$ increase and reach peak levels during the first $8 \mathrm{~h}$ of development, followed by a decline throughout the remaining stages of development. Conversely, culA rises in expression throughout development and reaches its peak level after $20 \mathrm{~h}$. Consistent with this, Mohanty et al. (2001) used an anti-CUL1 antibody to show that a cullin (presumed to be CulA) reaches peak levels after $16-20 \mathrm{~h}$ of development. Combined, these findings suggest that cullins may have specific roles during D. discoideum development. 


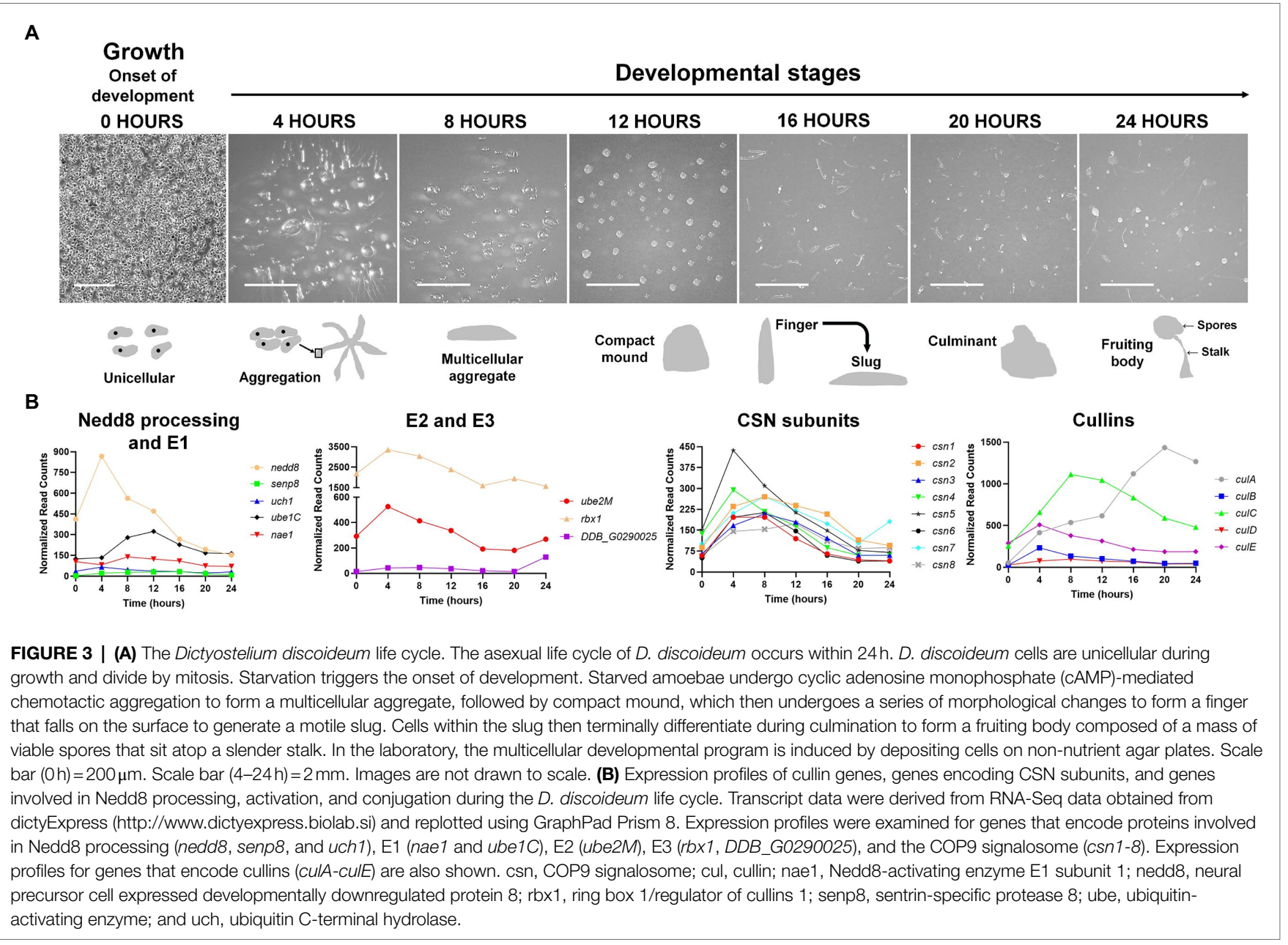

\section{The CulE Interactome in Dictyostelium discoideum}

In Sheikh et al. (2015) identified the SCF interactome in $D$. discoideum by expressing FLAG-tagged CulE in amoebae (Figure 5). In the interactome, proteins orthologous to components of the mammalian SCF complex were identified, such as homologs of SKP1 and three F-box proteins (FbxD, uncharacterized protein DDB0306343, and uncharacterized protein DDB0237864). Using an antibody directed against D. discoideum Skp1, another co-immunoprecipitation was performed that identified CulE as a Skp1-interactor via Western blotting (Sheikh et al., 2015). F-box proteins have been shown to interact with cullins in D. discoideum (Mohanty et al., 2001; Sheikh et al., 2015). For example, FbxD has been identified as a CulE interactor (discussed above; Sheikh et al., 2015; Figure 5). In addition, Mohanty et al. (2001) reported an in vivo interaction between CulA and FbxA using an antibody against human CUL1 that failed to detect a protein in $\mathrm{culA}^{-}$ cells via Western blotting. However, since the antibody was not specific for CulA, and there is strong sequence similarity between cullins in D. discoideum (and humans; Table 2; Sheikh et al., 2015), it is possible that another cullin was detected in the FbxA pull-down. Nonetheless, these observations suggest that the SCF complex associates with distinct cullins at different points in the life cycle to regulate specific processes during multicellular development. In addition, Skp1 modification affects the representation of F-box proteins in the Skp1 interactome suggesting that it influences the recruitment of $\mathrm{F}$ box proteins to the SCF complex (Sheikh et al., 2015).

The CulE interactome also revealed that known mechanisms of SCF regulation are conserved in D. discoideum (Enchev et al., 2015). For example, an ortholog of mammalian CAND1 (Cand1) was identified as an interactor suggesting that the mechanisms regulating CRL assembly and disassembly are similar in $D$. discoideum to what is observed in other eukaryotes (Figure 5). Furthermore, CulE was shown to interact with several proteins involved in neddylation and deneddylation such as Nedd8, Rbx1, and all the Csn subunits (Sheikh et al., 2015). As additional support for the neddylation of CulE, Western blotting for the CulE protein shows two distinct protein bands (Sheikh et al., 2015). In total, accumulated evidence strongly supports that not only is the neddylation machinery conserved in D. discoideum, but it also dynamically regulates the assembly of SCF complexes in $D$. discoideum by acting on cullins. 
TABLE 2 | Sequence similarity between cullins in humans and Dictyostelium discoideum.

\begin{tabular}{|c|c|c|c|c|c|c|c|}
\hline $\begin{array}{l}\text { Human protein } \\
\text { (Uniprot ID) }\end{array}$ & Size (aa) & $\begin{array}{l}\text { Dictyostelium discoideum } \\
\text { protein (dictyBase gene ID) }\end{array}$ & Size (aa) & $\begin{array}{c}\text { Region of } \\
\text { similarity (aa) }\end{array}$ & Identities (\%)* & Positives (\%)** & E-value \\
\hline \multirow{7}{*}{ CUL1 (Q13616) } & \multirow{6}{*}{776} & CulA (DDB_G0291972) & 770 & 772 & 51 & 68 & 0 \\
\hline & & CulB (DDB_G0267384) & 771 & 726 & 38 & 61 & $1 E-136$ \\
\hline & & CulE (DDB_G0278991) & 750 & 783 & 31 & 54 & $1 E-104$ \\
\hline & & CulC (DDB_G0284903) & 769 & 801 & 30 & 50 & 6E-99 \\
\hline & & CulD (DDB_G0292794) & 802 & 801 & 28 & 47 & $4 \mathrm{E}-69$ \\
\hline & & Anapc2 (DDB_G0276377) & 907 & 226 & 22 & 41 & $6 \mathrm{E}-05$ \\
\hline & \multirow{6}{*}{745} & CulA (DDB_G0291972) & 770 & 774 & 37 & 57 & $1 E-135$ \\
\hline \multirow{5}{*}{ CUL2 (Q13617) } & & CulB (DDB_G0267384) & 771 & 777 & 35 & 57 & $1 E-131$ \\
\hline & & CulC (DDB_G0284903) & 769 & 780 & 29 & 51 & $1 \mathrm{E}-87$ \\
\hline & & CulE (DDB_G0278991) & 750 & 760 & 30 & 51 & $1 \mathrm{E}-83$ \\
\hline & & CulD (DDB_G0292794) & 802 & 780 & 25 & 47 & $3 E-63$ \\
\hline & & Anapc2 (DDB_G0276377) & 907 & 157 & 24 & 40 & 0.013 \\
\hline \multirow{6}{*}{ CUL3 (Q13618) } & \multirow{6}{*}{768} & CulC (DDB_G0284903) & 769 & 777 & 48 & 66 & 0 \\
\hline & & CulD (DDB_G0292794) & 802 & 790 & 34 & 54 & $1 E-113$ \\
\hline & & CulA (DDB_G0291972) & 770 & 725 & 32 & 52 & 2E-97 \\
\hline & & CulB (DDB_G0267384) & 771 & 795 & 29 & 51 & $2 E-86$ \\
\hline & & CulE (DDB_G0278991) & 750 & 704 & 28 & 47 & $2 \mathrm{E}-62$ \\
\hline & & Anapc2 (DDB_G0276377) & 907 & 224 & 22 & 38 & 0.25 \\
\hline \multirow{6}{*}{ CUL4A (Q13619) } & \multirow{6}{*}{759} & CulD (DDB_G0292794) & 802 & 763 & 46 & 64 & 0 \\
\hline & & CulC (DDB_G0284903) & 769 & 776 & 38 & 59 & $1 E-142$ \\
\hline & & CulA (DDB_G0291972) & 770 & 700 & 33 & 53 & $2 E-88$ \\
\hline & & CulB (DDB_G0267384) & 771 & 741 & 27 & 49 & $2 \mathrm{E}-71$ \\
\hline & & CulE (DDB_G0278991) & 750 & 611 & 30 & 50 & $2 \mathrm{E}-61$ \\
\hline & & Anapc2 (DDB_G0276377) & 907 & 177 & 25 & 40 & $2 \mathrm{E}-04$ \\
\hline \multirow{6}{*}{ CUL4B (Q13620) } & \multirow{6}{*}{913} & CulD (DDB_G0292794) & 802 & 761 & 48 & 65 & 0 \\
\hline & & CulC (DDB_G0284903) & 769 & 766 & 38 & 60 & 1E-139 \\
\hline & & CulA (DDB_G0291972) & 770 & 701 & 32 & 54 & 8E-88 \\
\hline & & CulB (DDB_G0267384) & 771 & 741 & 26 & 48 & 7E-67 \\
\hline & & CulE (DDB_G0278991) & 750 & 610 & 29 & 50 & $5 E-59$ \\
\hline & & Anapc2 (DDB_G0276377) & 907 & 180 & 27 & 40 & 9E-05 \\
\hline \multirow{5}{*}{ CUL5 (Q93034) } & \multirow{5}{*}{780} & CulA (DDB_G0291972) & 770 & 797 & 28 & 52 & $3 E-85$ \\
\hline & & CulB (DDB_G0267384) & 771 & 764 & 29 & 51 & $6 E-84$ \\
\hline & & CulE (DDB_G0278991) & 750 & 661 & 28 & 52 & $3 E-65$ \\
\hline & & CulC (DDB_G0284903) & 769 & 689 & 26 & 48 & $8 E-55$ \\
\hline & & CulD (DDB_G0292794) & 802 & 674 & 26 & 47 & $1 \mathrm{E}-39$ \\
\hline CUL7 (Q14999) & 1,698 & CulA (DDB_G0291972) & 770 & 358 & 21 & 37 & 0.54 \\
\hline CUL9 (Q8IWT3) & 2,517 & CulA (DDB_G0291972) & 770 & 391 & 23 & 39 & 4E-05 \\
\hline
\end{tabular}

BLASTp searches were performed using dictyBase. Human proteins were used as query sequences (E-value, 1; Matrix, BLOSUM62; Filter, no). Sheikh et al. (2015) performed a similar analysis to show that CUL1 is most similar to CUIA, CUIE, and CUIB, CUL3 is most similar to CuIC, and CUL4B is most similar to CuID.

* Exact amino acid match.

**Similar amino acid match (e.g., both polar)

\section{THE ROLES OF CULLINS AND THEIR REGULATION DURING DICTYOSTELIUM DISCOIDEUM GROWTH}

\section{Roles of Neddylation and the CSN in Cell Proliferation}

While the role of neddylation is well established for Opisthokonta (major clade containing both fungi and animals), until recently, it was unknown whether neddylation regulates the life cycle of organisms belonging to Amoebozoa (sister group to Opisthokonta; Lammer et al., 1998; Liakopoulos et al., 1998; Gan-Erdene et al., 2003; Mendoza et al., 2003; Wu et al., 2003; Shen et al., 2005; Chan et al., 2008; Shin et al., 2011). Recent work used the well-established NAE1 inhibitor, MLN4924 (Pevonedistat), to reveal the roles of neddylation during $D$. discoideum growth and multicellular development (Huber et al., 2021). In Opisthokonta, neddylation regulates cell cycle progression and consequently, cell proliferation (Lammer et al., 1998; Liakopoulos et al., 1998; Gan-Erdene et al., 2003; Mendoza et al., 2003; Wu et al., 2003; Shen et al., 2005; Chan et al., 2008; Shin et al., 2011). In $D$. discoideum, MLN4924 significantly reduces cell proliferation in a dose-dependent manner during the growth phase of the life cycle (Figure 6; Huber et al., 2021). In addition, counting factor-associated protein $\mathrm{D}(\mathrm{CfaD})$, which is a secreted quorum sensing protein that modulates cell proliferation, was detected in the FbxD interactome (Figure 6; Bakthavatsalam et al., 2008; Sheikh et al., 2015). These findings indicate a conserved role for neddylation in regulating cell proliferation and support recent work linking neddylation to 


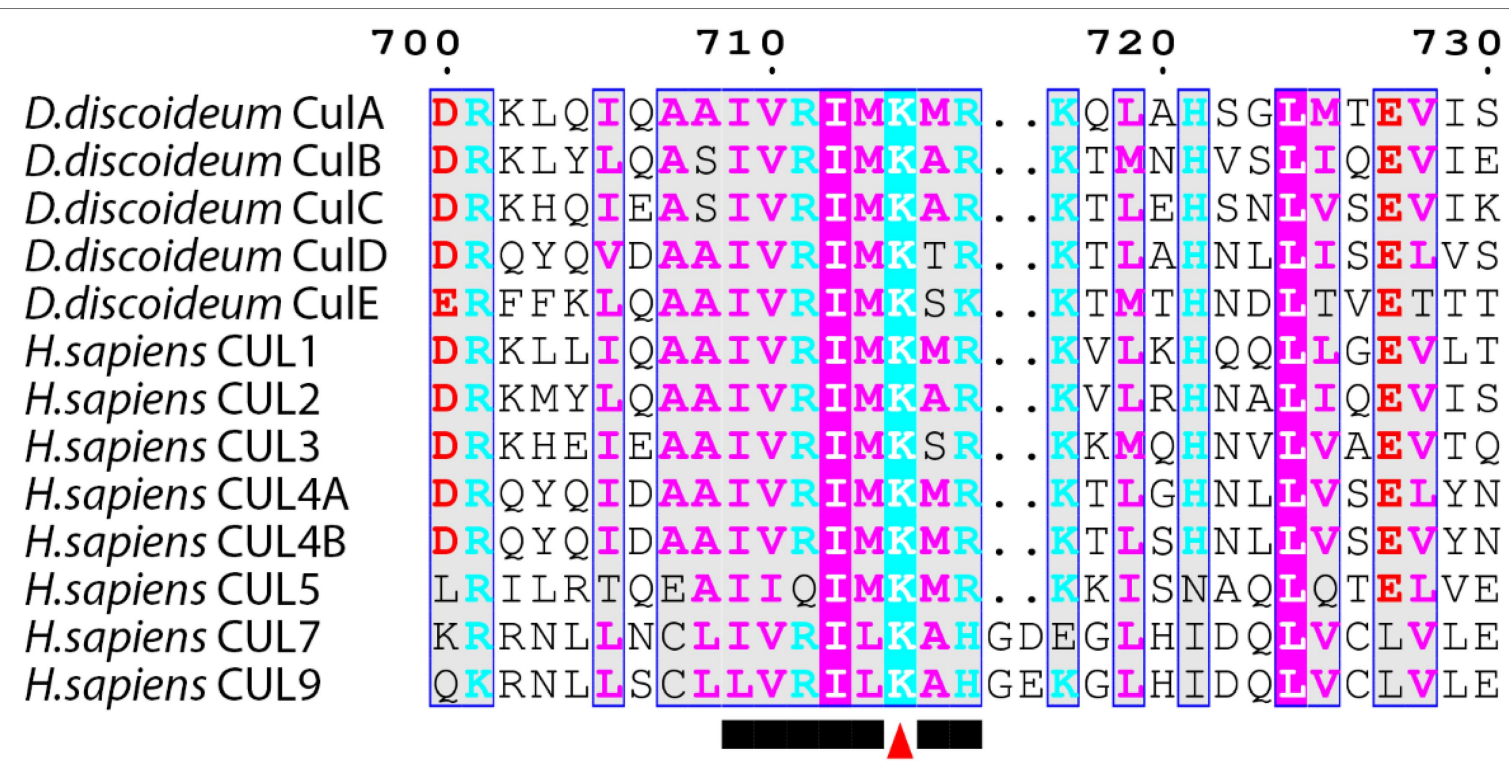

FIGURE 4 | Alignment of cullins from Dictyostelium discoideum and human. Sequences were aligned on MEGA7 using the MUSCLE alignment and displayed using ESPript 3.0. Colored residues indicate physiochemical properties and conservation at a position. Arrowhead marks the lysine motif where the NEDD8 protein is conjugated within the conserved motif.

the proliferation of cancer cells (Du et al., 2021; Zhang et al., 2021). However, whether MLN4924 specifically affects CRLs in $D$. discoideum remains to be determined since non-cullin targets of neddylation have been reported in several organisms including S. pombe, A. thaliana, and Trypanosoma brucei (Girdwood et al., 2012; Enchev et al., 2015; Mergner et al., 2015; Liao et al., 2017).

Deneddylation of cullins occurs through the actions of SENP8 and the CSN. Deneddylation by the CSN occurs via the CSN5 subunit, which is a metalloprotease (Cope et al., 2002; Echalier et al., 2013). For that reason, CSN5 has the highest selection pressure and is the most conserved of all the CSN subunits. Thus, in D. discoideum, Csn5 would be considered the essential subunit of the CSN required for the deneddylation of cullins. Consistent with this, loss of $\operatorname{csn} 5$ impairs cell proliferation in D. discoideum (Figure 6; Rosel and Kimmel, 2006). Since reduced neddylation and impaired deneddylation both impact cell proliferation $D$. discoideum, these findings indicate that cell proliferation in D. discoideum requires efficient cycling of neddylation and deneddylation.

\section{Role of Neddylation in Folic Acid-Mediated Chemotaxis}

D. discoideum cells use chemoattractants to sense nutrient levels within the environment. During the growth or feeding stage of the life cycle, D. discoideum cells sense and undergo chemotaxis towards folic acid, which is a biomolecule secreted by bacteria (Gerisch, 1982). Our group observed reduced chemotaxis toward folic acid when cells were treated with MLN4924 (Figure 6; Huber et al., 2021). Intriguingly, recent work supports a role for neddylation in regulating macrophage chemotaxis in chronic pancreatitis (Lin et al., 2021). Therefore, work in D. discoideum and humans indicates that neddylation plays an important regulatory role in chemotaxis. As a result, further probing of the chemotactic pathway in $D$. discoideum may improve our understanding of the mechanisms that CRLs use to regulate chemotaxis in humans.

\section{THE ROLE OF CULLINS AND THEIR REGULATION DURING DICTYOSTELIUM DISCOIDEUM EARLY DEVELOPMENT}

\section{CulA Is Required for cAMP-Mediated Chemotaxis}

During the early stages of multicellular development $(0-10 \mathrm{~h})$, $D$. discoideum amoebae aggregate by chemotaxis toward cyclic adenosine monophosphate (cAMP), which is produced and secreted by starving cells (Figure 3A; Almeida and Dilao, 2016; Nakajima and Sawai, 2016). Intriguingly, several cullin genes increase their expression during this stage of the life cycle (Figure 3B). Consistent with this, loss of $c u l A$ delays aggregation (Figure 6; Mohanty et al., 2001). Using an assay that examines chemotaxis competence, Mohanty et al. (2001) showed that the response of $c u l A^{-}$cells to cAMP was suboptimal suggesting that CulA has an early role in development by influencing cAMP-mediated chemotaxis during aggregation (Noegel et al., 1986). As discussed above, the role of neddylation in regulating chemotaxis in $D$. discoideum is consistent with its role in regulating macrophage chemotaxis in chronic pancreatitis (Lin et al., 2021). 


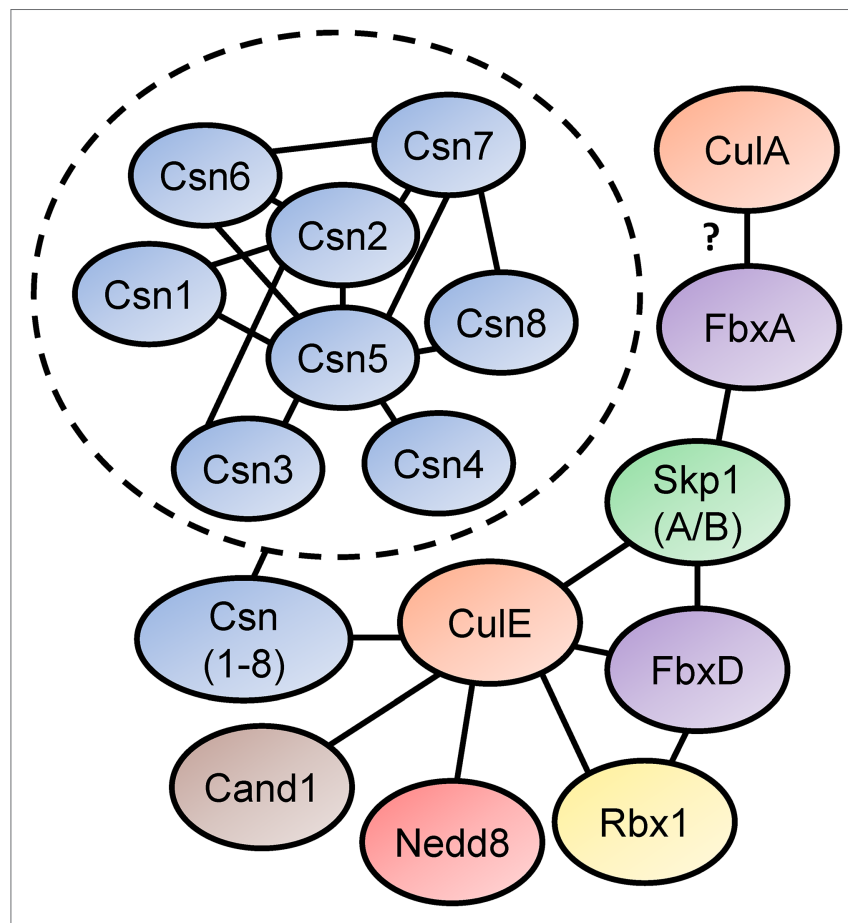

FIGURE 5 | Skp1-Cullin-F-box (SCF) complex interactome reveals components of the neddylation pathway and subunits of the CSN. Sheikh et al. (2015) identified proteins that interact with D. discoideum Skp1, FbxD, and CulE after performing co-immunoprecipitations for each protein. Interactors were identified using LC-MS/MS. Connecting lines indicate the interactions between the proteins. Mohanty et al. (2001) reported an interaction between a Dictyostelium discoideum cullin (presumably CulA) and FbxA. In the dashed circle, CSN subunit interactions with one another are shown. This was determined in a yeast two-hybrid screen by Rosel and Kimmel (2006). Cand1, cullin-associated Nedd8-dissociated protein 1; Csn, COP9 signalosome; Cul, cullin; Fbx, F-box protein; Nedd8, neural precursor cell expressed developmentally downregulated protein 8; Rbx1, ring box 1/ regulator of cullins 1; and Skp1, S phase kinase-associated protein 1.
In $D$. discoideum, cAMP controls the actions of cAMPdependent protein kinase A (PKA), which is a signaling kinase that regulates the expression of genes required for inducing aggregation, cell-type differentiation, and culmination (Loomis, 1998). Upstream of PKA activation, the cAMP phosphodiesterase, RegA, catalyzes the conversion of cAMP to $5^{\prime}$-adenosine monophosphate to prevent PKA activation (Shaulsky et al., 1996, 1998; Thomason et al., 1998). As a result of its regulatory role during aggregation, the level of RegA protein peaks at this stage of the developmental program (Mohanty et al., 2001). However, loss of culA or $f b x A$ causes the level of RegA protein to remain high even after aggregation (Mohanty et al., 2001; Tekinay et al., 2003). When culA- cells express a constitutively active PKA protein, the observed defects in aggregation and chemotaxis are absent (Mohanty et al., 2001). Since constitutively active PKA functions irrespective of cAMP levels, this supports the notion that abnormally high amounts of RegA interfere with cAMP levels to dysregulate PKA activity (Zhang et al., 2003). In conclusion, there is a significant role for CulA, and the SCF complex it functions with, in early development where it regulates the ubiquitination and degradation of RegA to maintain intracellular cAMP levels for PKA activation.

The SCF-dependent ubiquitination of RegA also appears to be dependent on extracellular signal-regulated kinase (ErkA), an ortholog of mammalian mitogen-activated protein kinase (MAPK). In D. discoideum, ErkA phosphorylates RegA to inhibit its activity and this phosphorylation could then prime RegA for SCF-mediated ubiquitination of RegA (Maeda et al., 2004; Kuburich et al., 2019). Additional work is required to determine if ErkA also directly regulates components of the SCF complex in $D$. discoideum. Intriguingly, ectopic expression of mouse Cul1 restored RegA degradation in $\mathrm{culA}^{-}$cells suggesting that the functions of mouse CUL1 and D. discoideum CulA are conserved (Mohanty et al., 2001).

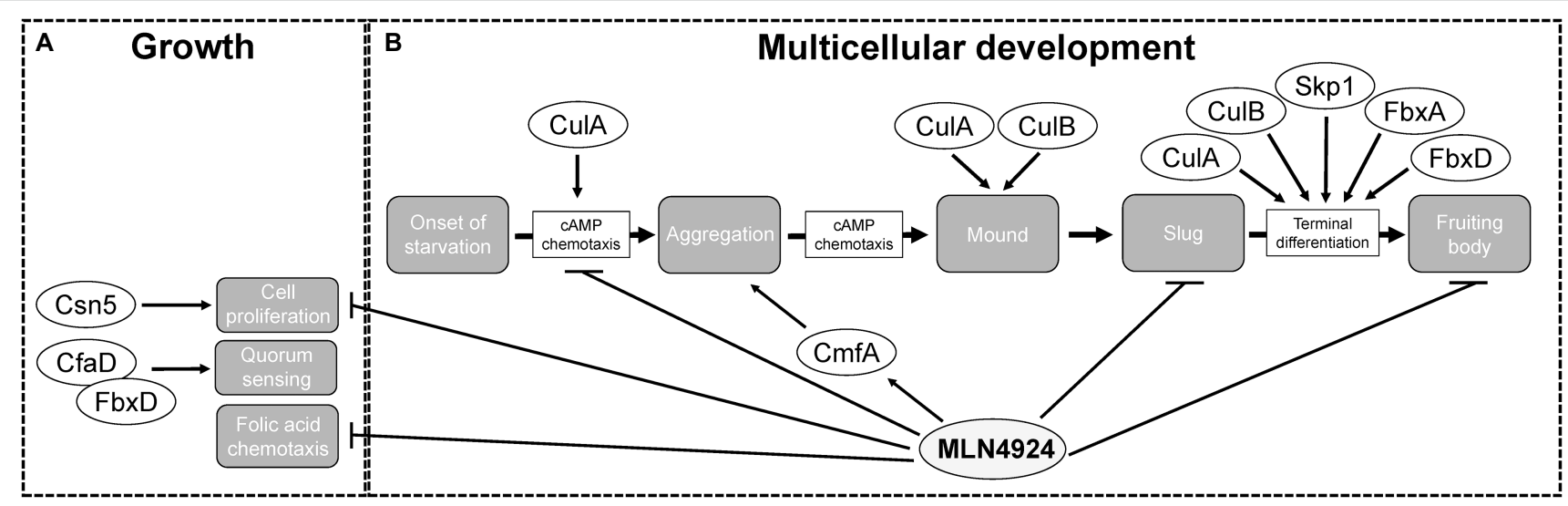

FIGURE 6 | The roles of cullins, neddylation, and the CSN during the Dictyostelium discoideum life cycle. (A) During growth, MLN4924 inhibits cell proliferation and folic acid-mediated chemotaxis. Loss of csn5 also inhibits cell proliferation. FbxD binds CfaD, which plays a role in quorum sensing. (B) During multicellular development, MLN4924 and loss of CuIA inhibit cAMP-mediated chemotaxis, which delays aggregation. MLN4924 also affects the intracellular and extracellular levels of $\mathrm{Cmf} A$, which plays a role in quorum sensing during aggregation. cul $A^{-}$and cul $B^{-}$cells form multi-tipped mounds during development, which is characteristic of $D$. discoideum mutants with defects in the autophagy pathway. MLN4924 inhibits slug and fruiting body formation. CulA, CulB, Skp1, FbxA, and FbxD collectively regulate fruiting body formation. CfaD, counting factor-associated protein $\mathrm{A} ; \mathrm{CmfA}$, conditioned media factor A; Csn5 and COP9 signalosome complex subunit 5; Cul, cullin; Fbx, F-box protein; and Skp1, S phase kinase-associated protein 1. 


\section{Role of Neddylation During Aggregation}

During $D$. discoideum development, cullins are differentially regulated and this is possibly linked to their roles in developmental processes at specific time points. Most cullin genes (except culA) peak in expression during the first $12 \mathrm{~h}$ of development when cells aggregate to form multicellular mounds (Figure 3B). In addition, genes involved in the conjugation of Nedd8 to cullins such as nedd8, uch1, nae1, ube $1 C$, ube $2 M$, and $r b x 1$ all follow a similar pattern of expression to the cullin genes, where they peak in expression during the first $12 \mathrm{~h}$ of development and then decline in expression throughout the remaining stages (Figure 3B). These data suggest that transcriptional changes in genes associated with neddylation occur in tandem with changes in the expression of cullin genes. Since neddylation promotes CRL assembly, this would allow for timely assembly of CRLs early in development to mediate the initiation of development and aggregation. In addition, CRL assembly and disassembly are thought to occur at least as frequently as new substrate selection (Kleiger et al., 2009; Deol et al., 2019). Consistent with this, the CSN subunits in D. discoideum also peak in expression during the first $12 \mathrm{~h}$ of development (Figure 3B). Combined, these results indicate that CRL assembly and disassembly are regulated by cycles of neddylation and deneddylation and that this cycling plays an important role in regulating the early stages of $D$. discoideum development.

Treatment of $D$. discoideum cells with MLN4924 delayed aggregation in a dose-dependent manner (Figure 6; Huber et al., 2021). Moreover, this delay persisted even after $24 \mathrm{~h}$, where cells were still unable to form compact mounds (Huber et al., 2021). Similarly, loss of culA was shown to impact cAMP-mediated chemotaxis and delay aggregation (Figure 6; Mohanty et al., 2001). Since neddylation inhibition phenocopies this result, it suggests that SCF complex assembly is inhibited when neddylation does not occur, which is consistent with observations in human cells (Zheng et al., 2002; Dubiel et al., 2013). These results also reveal that by upregulating the expression of nedd8 and cullins during cell aggregation, $D$. discoideum utilizes neddylation as a mechanism to facilitate the timely assembly of the SCF complex. Therefore, the mechanism underlying how neddylation influences cell aggregation is likely tied to its regulation of the SCF complex. Finally, MLN4924 also affects the secretion of the quorum sensing protein conditioned medium factor A ( CmfA) during aggregation (Figure 6; Huber et al., 2021). Upon starvation, amoebae begin secreting CmfA, which acts as a trigger for gene expression, as a high density of starved cells correlates to a high concentration of CmfA (Loomis, 2014). Once a CmfA threshold is reached, cells upregulate the expression of developmental genes, including spore coat protein $(\cot B)$ and cysteine protease $\mathrm{D}(\operatorname{cpr} D)$, and aggregate through cAMP-mediated chemotaxis (Loomis, 2014). Inhibition of neddylation by MLN4924 increases the intracellular and extracellular amounts of CmfA (Huber et al., 2021). While CmfA was not identified as an interactor of the $D$. discoideum SCF complex (Sheikh et al., 2015), the effect of MLN4924 on its intracellular and extracellular levels suggests it is regulated by neddylation.

\section{THE ROLES OF CULLINS AND THEIR REGULATION DURING THE MID-TO- LATE STAGES OF DICTYOSTELIUM DISCOIDEUM DEVELOPMENT}

\section{Roles of Cullins and Their Regulation During Slug Formation and Migration}

A potential explanation for how cullins influence cell-type differentiation involves the process of autophagy. Autophagy is a metabolic pathway that degrades intracellular material through lysosomal digestion (Mizushima et al., 2008). Autophagy is a required pathway for $D$. discoideum development and influences cell differentiation (Otto et al., 2003). In mammalian cells, the roles of cullins in regulating autophagy are wellestablished and occur at different steps in the autophagy pathway (McEwan and Dikic, 2014; Mesquita et al., 2017). In general, cullins regulate the ubiquitination of proteins belonging to the autophagy machinery, mTOR activation, and the activation transcription factors.

There also appears to be a link between cullins and autophagy in $D$. discoideum. During the transition from mounds to slugs, mounds form a single tip (Figure 3A). However, culA $A^{-}$and $c u l B^{-}$cells form multi-tipped mounds during development, which is characteristic of $D$. discoideum mutants with defects in the autophagy pathway (Figure 6; Mohanty et al., 2001; Wang and Kuspa, 2002; Otto et al., 2003; Mesquita et al., 2017). These results indicate that cullins may influence autophagy in $D$. discoideum thereby impacting cell differentiation. However, at present, this link remains to be experimentally determined for the $D$. discoideum cullins.

\section{Role of the SCF Complex in Oxygen Sensing}

Chemical and physical cues from the environment (e.g., light, temperature, and ammonia) are critical for regulating $D$. discoideum development, an organism normally found in soils worldwide (Bonner and Lamont, 2005; Singleton et al., 2006; West et al., 2007; Mathavarajah et al., 2017). Accessible oxygen influences slug polarity and migration, cell differentiation, and other aspects of multicellular development (Sternfeld and Bonner, 1977; Sternfeld and David, 1981; Sternfeld, 1988; Sawada et al., 1998; Bonner, 2003; Biondo et al., 2021; Cochet-Escartin et al., 2021). However, the way this oxygen is sensed by $D$. discoideum differs from what occurs in metazoans. Before the evolution of metazoans, single-celled eukaryotes like $D$. discoideum and Toxoplasma gondii lacked the hypoxia inducible factor system for oxygen sensing (Liu, 2017). Instead, these protozoans relied on modifying the SCF complex to mediate oxygen sensing and the mechanism behind this has been well studied in $D$. discoideum (reviewed in West et al., 2010). In D. discoideum, regulation of the SCF complex allows for oxygen sensing and this occurs via post-translational modifications. Skp1 of the SCF complex is post-translationally modified at Pro143 through prolyl hydroxylation (the addition of a hydroxyl 
group) via the prolyl hydroxylase gene ( $p h y A$; West et al., 2010). Since phyA is a direct oxygen sensor, the presence of oxygen functions as the initial stimuli for Skp1 hydroxylation. Following hydroxylation, Skp1 is O-glycosylated through the actions of several glycosyltransferases including GlcNAc transferase (GntA), poly-glycosyltransferase (PgtA), and alphagal-transferase (AgtA; in that order; Teng-Umnuay et al., 1999; van der Wel et al., 2001; Ketcham et al., 2004). Glycosylation then promotes the association of Skp1 with F-box proteins and allows for the rapid assembly of the SCF complex to regulate culmination and spore formation, and perhaps other oxygen-dependent developmental pathways (Xu et al., 2012; Sheikh et al., 2015). In conclusion, this represents a novel post-translational modification of the SCF complex that is utilized for oxygen-dependent development in $D$. discoideum.

\section{Cullins and Neddylation Influence Cell-Type Differentiation}

After aggregation, $D$. discoideum cells undergo cell-type differentiation to become pre-spore or pre-stalk cells, which each express cell-specific markers; extracellular matrix protein A (EcmA) for pre-stalk; and spore coat protein 60 (SP60/ CotC) for pre-spore (Fosnaugh and Loomis, 1989; Morrison et al., 1994; Williams, 2006). When these markers were examined post-aggregation in $\mathrm{culA}^{-}$cells, ecmA expression was absent and $\cot C$ expression was decreased (Mohanty et al., 2001). In addition, an altered ratio of pre-stalk to pre-spore cells was reported in $f b x A^{-}$cells and overexpression of FLAG-tagged FbxD has been shown to delay fruiting body formation (Nelson et al., 2000; Sheikh et al., 2015). Finally, inhibiting neddylation with MLN4924 was shown to impair fruiting body formation (Huber et al., 2021). Collectively, these findings indicate that $\mathrm{CulA}, \mathrm{FbxA}, \mathrm{FbxD}$, and neddylation have roles in cell differentiation.

Cullin genes in $D$. discoideum have selective roles in cell differentiation. Unlike what was seen with loss of $c u l A, c u l B^{-}$ cells express ecmA precociously and have a propensity to form pre-stalk cells (Wang and Kuspa, 2002). Moreover, culB- cells that express constitutively active PKA differentiate into stalk cells prior to even reaching the mound stage of development (Wang and Kuspa, 2002). In addition, PKA activity is antagonistic with the loss of $c u l B$, worsening the phenotype, and contrasting work with culA $^{-}$cells (Wang and Kuspa, 2002). These results suggest that CulB has non-CRL differentiation functions or is utilized in another unique CRL complex involved in regulating differentiation in $D$. discoideum.

Previous work suggests that cell-type differentiation in $D$. discoideum may also be influenced by culD and culE. In a recent study examining the expression of genes in different cell-types after differentiation, culD and culE transcripts were both preferentially upregulated in spores and downregulated in stalk cells (Kin et al., 2018). Altered expression in specific cell types may occur to (1) directly facilitate the terminal differentiation (i.e., pre-stalk to stalk cell) or (2) allow for functions related to the distinct roles of the cell types. Future work examining these two cullin genes will help elucidate how culD and culE contribute to cell differentiation during multicellular development.

\section{CONCLUSION}

D. discoideum is a well-established model organism that has been studied for close to 100 years (Raper, 1935). Its 24-h life cycle is comprised of unicellular and multicellular phases that allows for a detailed examination of a multitude of fundamental cellular and developmental processes in the context of a whole organism (Mathavarajah et al., 2017). D. discoideum can be cultured rapidly and inexpensively at room temperature in liquid medium (8-12 h doubling time) or on non-nutrient agar with bacteria (3-4h doubling time; Fey et al., 2007). Importantly, $D$. discoideum is genetically tractable and a variety of expression constructs have been generated to facilitate studies on protein localization and function (Levi et al., 2000; Kuspa, 2006; Veltman et al., 2009; Faix et al., 2013; Müller-Taubenberger and IshikawaAnkerhold, 2013; Friedrich et al., 2015; Yamashita et al., 2021). For these and other reasons, it has also been used as a highthroughput biomedical model for studying variety of human diseases (Huber, 2021; Kirolos et al., 2021; Mathavarajah et al., 2021; Pain et al., 2021).

Regulated protein degradation is an essential process in all eukaryotes. In $D$. discoideum, CRL-mediated ubiquitination regulates complex processes associated with growth and multicellular development (Figure 6). In addition, inhibiting neddylation with MLN4924 impacts cell proliferation, chemotaxis, aggregation, and multicellular development. As a result, future work in $D$. discoideum has the potential to enhance our understanding of the cellular and developmental roles of cullins, neddylation, and the CSN. For example, cell migration is an important physiological process that occurs during wound healing, embryonic development, and disease (e.g., cancer metastasis). D. discoideum is an ideal model system for studying fundamental aspects of cellular migration, particularly the mechanisms underlying chemotaxis (Kamimura and Ueda, 2021; Kuhn et al., 2021; Xu et al., 2021). As discussed in this review, neddylation regulates the migration of a variety of cell types (Park et al., 2018; Kim et al., 2021). Since MLN4924 inhibits cell migration during $D$. discoideum development, and CulA plays an important role in cAMP-mediated chemotaxis, $D$. discoideum can be used to further explore the role of neddylation and cullins in regulating cell migration and chemotaxis in normal and diseased cells. In addition, neddylation has been linked to autophagy regulation in esophageal and liver cancer (Luo et al., 2012; Chen et al., 2015; Liang et al., 2020). Since the mechanisms regulating autophagy in $D$. discoideum are like those that regulate autophagy in mammals (Mesquita et al., 2017), D. discoideum can be used to increase our understanding of how neddylation regulates autophagy. Finally, although there are significant differences between metazoan and $D$. discoideum development, studying the roles of neddylation and cullins in regulating multicellular development 
in $D$. discoideum may uncover conserved developmental roles that can then be validated in mammalian models and humans. This is important since there is a need to better understand the mechanisms regulating timely protein degradation events during metazoan development, as recent studies have reported an essential role for neddylation in cardiac development (Li et al., 2020). Thus, D. discoideum can be used as a model system to better understand different aspects of the neddylation pathway and CSN during development, which has implications for several diseases revolving around dysregulated neddylation. Together, this review highlights the use of $D$. discoideum as a model system to better understand the conserved cellular and developmental roles of cullins, neddylation, and the CSN.

\section{REFERENCES}

Almeida, S., and Dilao, R. (2016). Directional sensing and streaming in Dictyostelium aggregation. Phys. Rev. E 93:052402. doi: 10.1103/PhysRevE.93.052402

Andrews, P., He, Y. J., and Xiong, Y. (2006). Cytoplasmic localized ubiquitin ligase cullin 7 binds to p53 and promotes cell growth by antagonizing p53 function. Oncogene 25, 4534-4548. doi: 10.1038/sj.onc.1209490

Ardley, H. C., and Robinson, P. A. (2005). E3 ubiquitin ligases. Essays Biochem. 41, 15-30. doi: 10.1042/bse0410015

Bakthavatsalam, D., Brock, D. A., Nikravan, N., Houston, K. D., Hatton, R. D., and Gomer, R. H. (2008). The secreted Dictyostelium protein $\mathrm{CfaD}$ is a chalone. J. Cell Sci. 121, 2473-2480. doi: 10.1242/jcs.026682

Barth, E., Hübler, R., Baniahmad, A., and Marz, M. (2016). The evolution of COP9 signalosome in unicellular and multicellular organisms. Genome Biol. Evol. 8, 1279-1289. doi: 10.1093/gbe/evw073

Biondo, M., Panuzzo, C., Ali, S. M., Bozzaro, S., Osella, M., Bracco, E., et al. (2021). The dynamics of aerotaxis in a simple eukaryotic model. Front. Cell Dev. Biol. 9:720623. doi: 10.3389/fcell.2021.720623

Bohnsack, R. N., and Haas, A. L. (2003). Conservation in the mechanism of Nedd8 activation by the human AppBp1-Uba3 heterodimer. J. Biol. Chem. 278, 26823-26830. doi: 10.1074/jbc.M303177200

Bonner, J. T. (1949). The social amoebae. Sci. Am. 180, 44-47. doi: 10.1038/ scientificamerican0649-44

Bonner, J. T. (2003). Evolution of development in the cellular slime molds. Evol. Dev. 5, 305-313. doi: 10.1046/j.1525-142X.2003.03037.x

Bonner, J. T., and Lamont, D. S. (2005). Behavior of cellular slime molds in the soil. Mycologia 97, 178-184. doi: 10.3852/mycologia.97.1.178

Bosu, D. R., and Kipreos, E. T. (2008). Cullin-RING ubiquitin ligases: global regulation and activation cycles. Cell Div 3:7. doi: 10.1186/1747-1028-3-7

Braus, G. H., Irniger, S., and Bayram, Ö. (2010). Fungal development and the COP9 signalosome. Curr. Opin. Microbiol. 13, 672-676. doi: 10.1016/j. mib.2010.09.011

Brenner, M. (1977). Cyclic AMP gradient in migrating pseudoplasmodia of the cellular slime mold Dictyostelium discoideum. J. Biol. Chem. 252, 4073-4077. doi: 10.1016/S0021-9258(17)40234-1

Busch, S., Eckert, S. E., Krappmann, S., and Braus, G. H. (2003). The COP9 signalosome is an essential regulator of development in the filamentous fungus Aspergillus nidulans. Mol. Microbiol. 49, 717-730. doi: 10.1046/j.1365-2958.2003.03612.x

Cappadocia, L., and Lima, C. D. (2018). Ubiquitin-like protein conjugation: structures, chemistry, and mechanism. Chem. Rev. 118, 889-918. doi: 10.1021/ acs.chemrev.6b00737

Chan, Y., Yoon, J., Wu, J.-T., Kim, H.-J., Pan, K.-T., Yim, J., et al. (2008). DEN1 deneddylates non-cullin proteins in vivo. J. Cell Sci. 121, 3218-3223. doi: $10.1242 /$ jcs.030445

Chen, P., Hu, T., Liang, Y., Jiang, Y., Pan, Y., Li, C., et al. (2015). Synergistic inhibition of autophagy and neddylation pathways as a novel therapeutic approach for targeting liver cancer. Oncotarget 6, 9002-9017. doi: 10.18632/ oncotarget.3282

\section{AUTHOR CONTRIBUTIONS}

SM and RJH: conceptualization. WDK and SM: writing-original draft. WDK, SM, and RJH: writing-review and editing. RJH: supervision and funding acquisition. All authors contributed to the article and approved the submitted version.

\section{FUNDING}

This work was supported by a Discovery Grant from the Natural Sciences and Engineering Research Council of Canada (RGPIN2018-04855 to RJH). WDK was supported by a Queen Elizabeth II Graduate Scholarship in Science and Technology.

Chen, Y., Neve, R. L., and Liu, H. (2012). Neddylation dysfunction in Alzheimer's disease. J. Cell. Mol. Med. 16, 2583-2591. doi: 10.1111/j.1582-4934.2012. 01604.x

Chen, Z.-H., and Schaap, P. (2016). Secreted cyclic di-GMP induces stalk cell differentiation in the eukaryote Dictyostelium discoideum. J. Bacteriol. 198, 27-31. doi: 10.1128/JB.00321-15

Chung, D., and Dellaire, G. (2015). The role of the COP9 signalosome and neddylation in DNA damage signaling and repair. Biomolecules 5, 2388-2416. doi: $10.3390 /$ biom 5042388

Cochet-Escartin, O., Demircigil, M., Hirose, S., Allais, B., Gonzalo, P., Mikaelian, I., et al. (2021). Hypoxia triggers collective aerotactic migration in Dictyostelium discoideum. elife 10:e64731. doi: 10.7554/eLife.64731

Cope, G. A., and Deshaies, R. J. (2006). Targeted silencing of Jab1/Csn5 in human cells downregulates SCF activity through reduction of F-box protein levels. BMC Biochem. 7:1. doi: 10.1186/1471-2091-7-1

Cope, G. A., Suh, G. S. B., Aravind, L., Schwarz, S. E., Zipursky, S. L., Koonin, E. V., et al. (2002). Role of predicted metalloprotease motif of Jab1/Csn5 in cleavage of Nedd8 from Cul1. Science 298, 608-611. doi: 10.1126/science. 1075901

de Bie, P., and Ciechanover, A. (2011). Ubiquitination of E3 ligases: self-regulation of the ubiquitin system via proteolytic and non-proteolytic mechanisms. Cell Death Differ. 18, 1393-1402. doi: 10.1038/cdd.2011.16

Deol, K. K., Lorenz, S., and Strieter, E. R. (2019). Enzymatic logic of ubiquitin chain assembly. Front. Physiol. 10:835. doi: 10.3389/fphys.2019.00835

Doronkin, S., Djagaeva, I., and Beckendorf, S. K. (2003). The COP9 signalosome promotes degradation of cyclin E during early Drosophila oogenesis. Dev. Cell 4, 699-710. doi: 10.1016/S1534-5807(03)00121-7

Du, M. G., Peng, Z. Q., Gai, W. B., Liu, F., Liu, W., Chen, Y. J., et al. (2021). The absence of PTEN in breast cancer is a driver of MLN4924 resistance. Front. Cell Dev. Biol. 9:667435. doi: 10.3389/fcell.2021.667435

Dubiel, D., Gierisch, M. E., Huang, X., Dubiel, W., and Naumann, M. (2013). CAND1-dependent control of cullin 1-RING Ub ligases is essential for adipogenesis. Biochim. Biophys. Acta 1833, 1078-1084. doi: 10.1016/j. bbamcr.2013.01.005

Duda, D. M., Borg, L. A., Scott, D. C., Hunt, H. W., Hammel, M., and Schulman, B. A. (2008). Structural insights into NEDD8 activation of cullinRING ligases: conformational control of conjugation. Cell 134, 995-1006. doi: 10.1016/j.cell.2008.07.022

Echalier, A., Pan, Y., Birol, M., Tavernier, N., Pintard, L., Hoh, F., et al. (2013). Insights into the regulation of the human COP9 signalosome catalytic subunit, CSN5/Jab1. Proc. Natl. Acad. Sci. U. S. A. 110, 1273-1278. doi: 10.1073/ pnas. 1209345110

Ehrentraut, S. F., Curtis, V. F., Wang, R. X., Saeedi, B. J., Ehrentraut, H., Onyiah, J. C., et al. (2016). Perturbation of neddylation-dependent NF-kB responses in the intestinal epithelium drives apoptosis and inhibits resolution of mucosal inflammation. Mol. Biol. Cell 27, 3687-3694. doi: 10.1091/mbc. E16-05-0273

Enchev, R. I., Schulman, B. A., and Peter, M. (2015). Protein neddylation: beyond cullin-RING ligases. Nat. Rev. Mol. Cell Biol. 16, 30-44. doi: 10.1038/ nrm3919 
Faix, J., Linkner, J., Nordholz, B., Platt, J. L., Liao, X. H., and Kimmel, A. R. (2013). The application of the Cre-loxP system for generating multiple knock-out and knock-in targeted loci. Methods Mol. Biol. 983, 249-267. doi: 10.1007/978-1-62703-302-2_13

Fey, P., Kowal, A. S., Gaudet, P., Pilcher, K. E., and Chisholm, R. L. (2007). Protocols for growth and development of Dictyostelium discoideum. Nat. Protoc. 2, 1307-1316. doi: 10.1038/nprot.2007.178

Forman, D., and Garrod, D. R. (1977). Pattern formation in Dictyostelium discoideum. II. Differentiation and pattern formation in non-polar aggregates. J. Embryol. Exp. Morpholog. 40, 229-243

Fosnaugh, K., and Loomis, W. F. (1989). Spore coat genes SP60 and SP70 of Dictyostelium discoideum. Mol. Cell. Biol. 9, 5215-5218. doi: 10.1128/ mcb.9.11.5215-5218.1989

Frickel, E.-M., Quesada, V., Muething, L., Gubbels, M.-J., Spooner, E., Ploegh, H., et al. (2007). Apicomplexan UCHL3 retains dual specificity for ubiquitin and Nedd8 throughout evolution. Cell. Microbiol. 9, 1601-1610. doi: 10.1111/j. 1462-5822.2007.00896.x

Friedrich, M., Meier, D., Schuster, I., and Nellen, W. (2015). A simple retroelement based knock-down system in Dictyostelium: further insights into RNA interference mechanisms. PLoS One 10:e0131271. doi: 10.1371/journal. pone. 0131271

Gan-Erdene, T., Nagamalleswari, K., Yin, L., Wu, K., Pan, Z.-Q., and Wilkinson, K. D. (2003). Identification and characterization of DEN1, a deneddylase of the ULP family. J. Biol. Chem. 278, 28892-28900. doi: 10.1074/ jbc.M302890200

Gaudet, P., Williams, J. G., Fey, P., and Chisholm, R. L. (2008). An anatomy ontology to represent biological knowledge in Dictyostelium discoideum. BMC Genomics 9:130. doi: 10.1186/1471-2164-9-130

Gerisch, G. (1982). Chemotaxis in Dictyostelium. Annu. Rev. Physiol. 44, 535-552. doi: 10.1146/annurev.ph.44.030182.002535

Gilberto, S., and Peter, M. (2017). Dynamic ubiquitin signaling in cell cycle regulation. J. Cell Biol. 216, 2259-2271. doi: 10.1083/jcb.201703170

Girdwood, D., Robertson, M., and Gordon, C. (2012). Constitutively active cullinRING-ligases fail to rescue loss of NEDD8 conjugation in Schizosaccharomyces pombe. FEBS Lett. 586, 1522-1528. doi: 10.1016/j.febslet.2012.04.011

Gong, L., and Yeh, E. T. (1999). Identification of the activating and conjugating enzymes of the NEDD8 conjugation pathway. J. Biol. Chem. 274, 12036-12042. doi: $10.1074 /$ jbc.274.17.12036

He, Q., Cheng, P., He, Q., and Lui, Y. (2005). The COP9 signalosome regulates the neurospora circadian clock by controlling the stability of the SCFFWD-1 complex. Genes Dev. 19, 1518-1531. doi: 10.1101/gad.1322205

Heidel, A. J., Lawal, H. M., Felder, M., Schilde, C., Helps, N. R., Tunggal, B., et al. (2011). Phylogeny-wide analysis of social amoeba genomes highlights ancient origins for complex intercellular communication. Genome Res. 21, 1882-1891. doi: 10.1101/gr.121137.111

Hemelaar, J., Borodovsky, A., Kessler, B. M., Reverter, D., Cook, J., Kolli, N., et al. (2004). Specific and covalent targeting of conjugating and deconjugating enzymes of ubiquitin-like proteins. Mol. Cell. Biol. 24, 84-95. doi: 10.1128/ MCB.24.1.84-95.2004

Huang, D. T., Ayrault, O., Hunt, H. W., Taherbhoy, A. M., Duda, D. M., Scott, D. C., et al. (2009). E2-RING expansion of the NEDD8 cascade confers specificity to cullin modification. Mol. Cell 33, 483-495. doi: 10.1016/j. molcel.2009.01.011

Huang, X., Ordemann, J., Pratschke, J., and Dubiel, W. (2016). Overexpression of COP9 signalosome subunits, CSN7A and CSN7B, exerts different effects on adipogenic differentiation. FEBS Open Bio. 6, 1102-1112. doi: 10.1002/2211-5463.12129

Huang, D. T., Paydar, A., Zhuang, M., Waddell, M. B., Holton, J. M., and Schulman, B. A. (2005). Structural basis for recruitment of Ubc12 by an E2 binding domain in NEDD8's E1. Mol. Cell 17, 341-350. doi: 10.1016/j. molcel.2004.12.020

Huber, R. J. (2021). Altered protein secretion in Batten disease. Dis. Model. Mech. 14:dmm049152. doi: 10.1242/dmm.049152

Huber, R. J., Kim, W. D., and Mathavarajah, S. (2021). Inhibiting neddylation with MLN4924 suppresses growth and delays multicellular development in Dictyostelium discoideum. Biomolecules 11:482. doi: 10.3390/biom11030482

Jin, L., Williamson, A., Banerjee, S., Philipp, I., and Rape, M. (2008). Mechanism of ubiquitin-chain formation by the human anaphase-promoting complex. Cell 133, 653-665. doi: 10.1016/j.cell.2008.04.012
Johnston, S. C., Riddle, S. M., Cohen, R. E., and Hill, C. P. (1999). Structural basis for the specificity of ubiquitin C-terminal hydrolases. EMBO J. 18, 3877-3887. doi: 10.1093/emboj/18.14.3877

Jones, J., Wu, K., Yang, Y., Guerrero, C., Nillegoda, N., Pan, Z.-Q., et al. (2008). A targeted proteomic analysis of the ubiquitin-like modifier nedd8 and associated proteins. J. Proteome Res. 7, 1274-1287. doi: 10.1021/pr700749v

Kamimura, Y., and Ueda, M. (2021). Different heterotrimeric G protein dynamics for wide-range chemotaxis in eukaryotic cells. Front. Cell Dev. Biol. 9:724797. doi: $10.3389 /$ fcell.2021.724797

Kamitani, T., Kito, K., Nguyen, H. P., and Yeh, E. T. (1997). Characterization of NEDD8, a developmentally down-regulated ubiquitin-like protein. J. Biol. Chem. 272, 28557-28562. doi: 10.1074/jbc.272.45.28557

Kamura, T., Conrad, M. N., Yan, Q., Conaway, R., and Conaway, J. W. (1999). The Rbx1 subunit of SCF and VHL E3 ubiquitin ligase activates Rub1 modification of cullins Cdc53 and Cul2. Genes Dev. 13, 2928-2933. doi: 10.1101/gad.13.22.2928

Kawakami, T., Chiba, T., Suzuki, T., Iwai, K., Yamanaka, K., Minato, N., et al. (2001). NEDD8 recruits E2-ubiquitin to SCF E3 ligase. EMBO J. 20, 4003-4012. doi: $10.1093 / \mathrm{emboj} / 20.15 .4003$

Ketcham, C., Wang, F., Fisher, S. Z., Ercan, A., van der Wel, H., Locke, R. D., et al. (2004). Specificity of a soluble UDP-galactose: fucoside $\alpha 1,3-$ galactosyltransferase that modifies the cytoplasmic glycoprotein Skp1 in Dictyostelium. J. Biol. Chem. 279, 29050-29059. doi: 10.1074/jbc.M313858200

Keuss, M. J., Hjerpe, R., Hsia, O., Gourlay, R., Burchmore, R., Trost, M., et al. (2019). Unanchored tri-NEDD8 inhibits PARP-1 to protect from oxidative stress-induced cell death. EMBO J. 38:e100024. doi: 10.15252/embj.2018100024

Kim, A. Y., Bommeljé, C. C., Lee, B. E., Yonekawa, Y., Choi, L., Morris, L. G., et al. (2008). SCCRO (DCUN1D1) is an essential component of the E3 complex for neddylation. J. Biol. Chem. 283, 33211-33220. doi: 10.1074/ jbc.M804440200

Kim, Y., Park, J. B., Fukuda, J., Watanabe, M., and Chun, Y. S. (2021). The effect of neddylation blockade on slug-dependent cancer cell migration is regulated by p53 mutation status. Cancer 13:531. doi: 10.3390/cancers13030531

Kin, K., Forbes, G., Cassidy, A., and Schaap, P. (2018). Cell-type specific RNASeq reveals novel roles and regulatory programs for terminally differentiated Dictyostelium cells. BMC Genomics 19:764. doi: 10.1186/s12864-018-5146-3

Kirolos, S. A., Rijal, R., Consalvo, K. M., and Gomer, R. H. (2021). Using Dictyostelium to develop therapeutics for acute respiratory distress syndrome. Front. Cell Dev. Biol. 9:710005. doi: 10.3389/fcell.2021.710005

Kleiger, G., Saha, A., Lewis, S., Kuhlman, B., and Deshaies, R. J. (2009). Rapid E2-E3 assembly and disassembly enable processive ubiquitylation of cullinRING ubiquitin ligase substrates. Cell 139, 957-968. doi: 10.1016/j. cell.2009.10.030

Kuburich, N. A., Adhikari, N., and Hadwiger, J. A. (2019). Multiple phosphorylation sites on the RegA phosphodiesterase regulate Dictyostelium development Cell. Signal. 57, 65-75. doi: 10.1016/j.cellsig.2019.02.005

Kuhn, J., Lin, Y., and Devreotes, P. N. (2021). Using live-cell imaging and synthetic biology to probe directed migration in Dictyostelium. Front. Cell Dev. Biol. 9:740205. doi: 10.3389/fcell.2021.740205

Kumar, S., Stecher, G., and Tamura, K. (2016). MEGA7: molecular evolutionary genetics analysis version 7.0 for bigger datasets. Mol. Biol. Evol. 33, 1870-1874. doi: 10.1093/molbev/msw054

Kuspa, A. (2006). Restriction enzyme-mediated integration (REMI) mutagenesis. Methods Mol. Biol. 346, 201-209. doi: 10.1385/1-59745-144-4:201

Lammer, D., Mathias, N., Laplaza, J. M., Jiang, W., Lui, Y., Callis, J., et al. (1998). Modification of yeast Cdc53p by the ubiquitin-related protein rublp affects function of the $\mathrm{SCF}^{\mathrm{Cdc} 4}$ complex. Genes Dev. 12, 914-926. doi: 10.1101/ gad.12.7.914

Levi, S., Polyakov, M., and Egelhoff, T. T. (2000). Green fluorescent protein and epitope tag fusion vectors for Dictyostelium discoideum. Plasmid 44, 231-238. doi: $10.1006 /$ plas.2000.1487

Li, J. M., and Jin, J. (2012). CRL ubiquitin ligases and DNA damage response. Front. Oncol. 2:29. doi: 10.3389/fonc.2012.00029

Li, J., Zou, J., Littlejohn, R., Liu, J., and Su, H. (2020). Neddylation, an emerging mechanism regulating cardiac development and function. Front. Physiol. 11:612927. doi: 10.3389/fphys.2020.612927

Liakopoulos, D., Doenges, G., Matuschewski, K., and Jentsch, S. (1998). A novel protein modification pathway related to the ubiquitin system. $E M B O$ J. 17, 2208-2214. doi: 10.1093/emboj/17.8.2208 
Liang, Y., Jiang, Y., Jin, X., Chen, P., Heng, Y., Cai, L., et al. (2020). Neddylation inhibition activates the protective autophagy through NF- $\kappa \mathrm{B}$-catalase-ATF3 Axis in human esophageal cancer cells. Cell Commun. Signal 18:72. doi: 10.1186/s12964-020-00576-Z

Liao, S., Hu, H., Wang, T., Tu, X., and Li, Z. (2017). The protein neddylation pathway in Trypanosoma brucei: functional characterization and substrate identification. J. Biol. Chem. 292, 1081-1091. doi: 10.1074/jbc.M116.766741

Lin, Y., Chen, Y., Feng, W., Hua, R., Zhang, J., Huo, Y., et al. (2021). Neddylation pathway alleviates chronic pancreatitis by reducing HIF1 $\alpha$-CCL5-dependent macrophage infiltration. Cell Death Dis. 12:273. doi: 10.1038/s41419-021-03549-3

Linghu, B., Callis, J., and Goebl, M. G. (2002). Rublp processing by Yuhlp is required for wild-type levels of Rublp conjugation to Cdc53p. Eukaryot. Cell 1, 491-494. doi: 10.1128/EC.1.3.491-494.2002

Liu, J., Furukawa, M., Matsumoto, T., and Xiong, Y. (2002). NEDD8 modification of CUL1 dissociates p120(CAND1), an inhibitor of CUL1-SKP1 binding and SCF ligases. Mol. Cell 10, 1511-1518. doi: 10.1016/s1097-2765(02)00783-9

Liu, T. (2017). Evolutionary insights into oxygen sensing. FASEB J. 31:606.1. doi: 10.1096/fasebj.31.1_supplement.606.1

Liu, X., Reitsma, J. M., Mamrosh, J. L., Zhang, Y., Straube, R., and Deshaies, R. J. (2018). Cand1-mediated adaptive exchange mechanism enables variation in F-box protein expression. Mol. Cell 69, 773-786. doi: 10.1016/j. molcel.2018.01.038

Lobato-Gil, S., Heidelberger, J. B., Maghames, C., Bailly, A., Brunello, L., Rodriguez, M. S., et al. (2021). Proteome-wide identification of NEDD8 modification sites reveals distinct proteomes for canonical and atypical NEDDylation. Cell Rep. 34:108635. doi: 10.1016/j.celrep.2020.108635

Loomis, W. F. (1998). Role of PKA in the timing of developmental events in Dictyostelium cells. Microbiol. Mol. Biol. Rev. 62, 684-694. doi: 10.1128/ MMBR.62.3.684-694.1998

Loomis, W. F. (2014). Cell signaling during development of Dictyostelium. Dev. Biol. 391, 1-16. doi: 10.1016/j.ydbio.2014.04.001

Luo, Z., Yu, G., Lee, H. W., Li, L., Wang, L., Yang, D., et al. (2012). The Nedd8-activating enzyme inhibitor MLN4924 induces autophagy and apoptosis to suppress liver cancer cell growth. Cancer Res. 72, 3360-3371. doi: 10.1158/0008-5472.CAN-12-0388

Maeda, M., Lu, S., Shaulsky, G., Miyazaki, Y., Kuwayama, K., Tnaka, Y., et al. (2004). Periodic signaling controlled by an oscillatory circuit that includes protein kinases ERK2 and PKA. Science 304, 875-878. doi: 10.1126/ science. 1094647

Maghames, C. M., Lobato-Gil, S., Perrin, A., Trauchessec, H., Rodriguez, M. S., Urbach, S., et al. (2018). Neddylation promotes nuclear protein aggregation and protects the ubiquitin proteasome system upon proteotoxic stress. Nat. Commun. 9:4376. doi: 10.1038/s41467-018-06365-0

Marée, A. F., and Hogeweg, P. (2001). How amoeboids self-organize into a fruiting body: multicellular coordination in Dictyostelium discoideum. Proc. Natl. Acad. Sci. U. S. A. 98, 3879-3883. doi: 10.1073/pnas.061535198

Mathavarajah, S., Flores, A., and Huber, R. J. (2017). Dictyostelium discoideum: a model system for cell and developmental biology. Curr. Protoc. Essent. Lab. Tech. 15, 14.1.1-14.1.19. doi: 10.1002/cpet.15

Mathavarajah, S., VanIderstine, C., Dellaire, G., and Huber, R. J. (2021). Cancer and the breakdown of multicellularity: what Dictyostelium discoideum, a social amoeba, can teach us. Bioessays 43:e2000156. doi: 10.1002/bies.202000156

McEwan, D. G., and Dikic, I. (2014). Cullins keep autophagy under control. Dev. Cell 31, 675-676. doi: 10.1016/j.devcel.2014.12.010

Mendoza, H. M., Shen, L.-N., Botting, C., Lewis, A., Chen, J., Ink, B., et al. (2003). NEDP1, a highly conserved cysteine protease that deneddylates cullins. J. Biol. Chem. 278, 25637-25643. doi: 10.1074/jbc.M212948200

Mergner, J., Heinzlmeir, S., Kuster, B., and Schwechheimer, C. (2015). DENEDDYLASE1 deconjugates NEDD8 from non-cullin protein substrates in Arabidopsis thaliana. Plant Cell 27, 741-753. doi: 10.1105/tpc.114.135996

Mesquita, A., Cardenal-Muñoz, E., Dominguez, E., Muñoz-Braceras, S., Nuñez-Corcuera, B., Philips, B. A., et al. (2017). Autophagy in Dictyostelium: mechanisms, regulation and disease in a simple biomedical model. Autophagy 13, 24-40. doi: 10.1080/15548627.2016.1226737

Mikus, P., and Zundel, W. (2005). COPing with hypoxia. Semin. Cell Dev. Biol. 16, 462-473. doi: 10.1016/j.semcdb.2005.03.002

Mizushima, N., Levine, B., Cuervo, A. M., and Klionsky, D. J. (2008). Autophagy fights disease through cellular self-digestion. Nature 451:1069. doi: 10.1038/ nature 06639
Mohanty, S., Lee, S., Yadava, N., Dealy, M. J., Johnson, R. S., and Firtel, R. A. (2001). Regulated protein degradation controls PKA function and cell-type differentiation in Dictyostelium. Genes Dev. 15, 1435-1448. doi: 10.1101/ $\operatorname{gad} .871101$

Morrison, A., Blanton, R. L., Grimson, M., Fuchs, M., Williams, K., and Williams, J. (1994). Disruption of the gene encoding the EcmA, extracellular matrix protein of Dictyostelium alters slug morphology. Dev. Biol. 163, 457-466. doi: 10.1006/dbio.1994.1162

Müller-Taubenberger, A., and Ishikawa-Ankerhold, H. C. (2013). Fluorescent reporters and methods to analyze fluorescent signals. Methods Mol. Biol. 983, 93-112. doi: 10.1007/978-1-62703-302-2 5

Nakajima, A., and Sawai, S. (2016). Dissecting spatial and temporal sensing in Dictyostelium chemotaxis using a wave gradient generator. Methods Mol. Biol. 1407, 107-122. doi: 10.1007/978-1-4939-3480-5_8

Nelson, M. K., Clark, A., Abe, T., Nomura, A., Yadava, N., Funair, C. J., et al. (2000). An F-box/WD40 repeat-containing protein important for Dictyostelium cell-type proportioning, slug behaviour, and culmination. Dev. Biol. 224, 42-59. doi: 10.1006/dbio.2000.9793

Noegel, A., Gerisch, G., Stadler, J., and Westpal, M. (1986). Complete sequence and transcript regulation of a cell adhesion protein from aggregating Dictyostelium cells. EMBO J. 5, 1473-1476. doi: 10.1002/j.1460-2075.1986. tb04384.x

Osaka, F., Kawasaki, H., Aida, N., Saeki, M., Chiba, T., Kawashima, S., et al. (1998). A new NEDD8-ligating system for cullin-4A. Genes Dev. 12, 2263-2268. doi: $10.1101 /$ gad.12.15.2263

Osaka, F., Saeki, M., Katayama, S., Aida, N., Toh-e, A., Kominami, K.-I., et al. (2000). Covalent modifier NEDD8 is essential for SCF ubiquitin-ligase in fission yeast. EMBO J. 19, 3475-3484. doi: 10.1093/emboj/19.13.3475

Otto, G. P., Wu, M. Y., Kazgan, N., Anderson, O. R., and Kessin, R. H. (2003). Macroautophagy is required for multicellular development of the social amoeba Dictyostelium discoideum. J. Biol. Chem. 278, 17636-17645. doi: 10.1074/jbc.M212467200

Pain, E., Shinhmar, S., and Williams, R. S. B. (2021). Using Dictyostelium to advance our understanding of the role of medium chain fatty acids in health and disease. Front. Cell Dev. Biol. 9:722066. doi: 10.3389/fcell.2021. 722066

Pan, Z. Q., Kentsis, A., Dias, D. C., Yamoah, K., and Wu, K. (2004). Nedd8 on cullin: building an expressway to protein destruction. Oncogene 23, 1985-1997. doi: 10.1038/sj.onc.1207414

Park, S. Y., Park, J. W., Lee, G. W., Li, L., and Chun, Y. S. (2018). Inhibition of neddylation facilitates cell migration through enhanced phosphorylation of caveolin-1 in PC3 and U373MG cells. BMC Cancer 18:30. doi: 10.1186/ s12885-017-3942-9

Petroski, M. D., and Deshaies, R. J. (2005). Function and regulation of cullinRING ubiquitin ligases. Nat. Rev. Mol. Cell Biol. 6, 9-20. doi: 10.1038/ nrm 1547

Pierce, N. W., Lee, J. E., Liu, X., Sweredoski, M. J., Graham, R. L., Larimore, E. A., et al. (2013). Cand1 promotes assembly of new SCF complexes through dynamic exchange of $\mathrm{F}$ box proteins. Cell 153, 206-215. doi: 10.1016/j. cell.2013.02.024

Pintard, L., Kurz, T., Glaser, S., Willis, J. H., Peter, M., and Bowerman, B. (2003). Neddylation and deneddylation of CUL-3 is required to target MEI-1/ Katanin for degradation at the meiosis-to-mitosis transition in C. elegans. Curr. Biol. 13, 911-921. doi: 10.1016/S0960-9822(03)00336-1

Qi, J., and Ronai, Z. A. (2015). Dysregulation of ubiquitin ligases in cancer. Drug Resist. Updat. 23, 1-11. doi: 10.1016/j.drup.2015.09.001

Rao, F., Lin, H., and Su, Y. (2020). Cullin-RING ligase regulation by the COP9 signalosome: structural mechanisms and new physiologic players. Adv. Exp. Med. Biol. 1217, 47-60. doi: 10.1007/978-981-15-1025-0_4

Raper, K. B. (1935). Dictyostelium discoideum, a new species of slime mold from decaying forest leaves. J. Agric. Res. 50, 135-147.

Raper, K. B. (1940). Pseudoplasmodium formation and organization in Dictyostelium discoideum. J. Elisha Mitch. Sci. Soc. 56, 241-282.

Robert, X., and Gouet, P. (2014). Deciphering key features in protein structures with the new ENDscript server. Nucleic Acids Res. 42, W320-W324. doi: $10.1093 / \mathrm{nar} / \mathrm{gku} 316$

Rosel, D., and Kimmel, A. R. (2006). The COP9 signalosome regulates cell proliferation of Dictyostelium discoideum. Eur. J. Cell Biol. 85, 1023-1034. doi: $10.1016 /$ j.ejcb.2006.04.006 
Saha, A., and Deshaies, R. J. (2008). Multimodal activation of the ubiquitin ligase SCF by Nedd8 conjugation. Mol. Cell 32, 21-31. doi: 10.1016/j.molcel.2008.08.021

Sarikas, A., Hartmann, T., and Pan, Z. Q. (2011). The cullin protein family. Genome Biol. 12:220. doi: 10.1186/gb-2011-12-4-220

Sawada, Y., Maeda, Y., Takeuchi, I., Williams, J., and Maeda, Y. (1998). Rapid patterning of Dictyostelium discoideum cells under confined geometry and its relation to differentiation. Develop. Growth Differ. 40, 113-120. doi: 10.1046/j.1440-169X.1998.t01-2-00013.x

Schmidt, M. W., McQuary, P. R., Wee, S., Hofmann, K., and Wolf, D. A. (2010). F-box-directed CRL complex assembly and regulation by the CSN and CAND1. Mol. Cell 35, 586-597. doi: 10.1016/j.molcel.2009.07.024

Schwechheimer, C., Serino, G., Callis, J., Crosby, W. L., Lyapina, S., Deshaies, R. J., et al. (2001). Interactions of the COP9 signalosome with the E3 ubiquitin ligase SCFTIRI in mediating auxin response. Science 292, 1379-1382. doi: 10.1126/science. 1059776

Schwechheimer, C., and Villalobos, L. I. A. C. (2004). Cullin-containing E3 ubiquitin ligases in plant development. Curr. Opin. Plant Biol. 7, 677-686. doi: $10.1016 /$ j.pbi.2004.09.009

Scott, D. C., Monda, J. K., Grace, C. R. R., Duda, D. M., Kriwacki, R. W., Kurz, T., et al. (2010). A dual E3 mechanism for Rub1 ligation to Cdc53. Mol. Cell 39, 784-796. doi: 10.1016/j.molcel.2010.08.030

Serrano, I., Campos, L., and Rivas, S. (2018). Roles of E3 ubiquitin-ligases in nuclear protein homeostasis during plant stress responses. Front. Plant Sci. 9:139. doi: $10.3389 /$ fpls.2018.00139

Shaulsky, G., Escalante, R., and Loomis, W. F. (1996). Developmental signal transduction pathways uncovered by genetic suppressors. Proc. Natl. Acad. Sci. U. S. A. 93, 15260-15265. doi: 10.1073/pnas.93.26.15260

Shaulsky, G., Fuller, D., and Loomis, W. F. (1998). A cAMP-phosphodiesterase controls PKA-dependent differentiation. Development 125, 691-699

Sheikh, M. O., Xu, Y., van der Wel, H., Walden, P., Hartson, S. D., and West, C. M. (2015). Glycosylation of Skp1 promotes formation of Skp1cullin-1-F-box protein complexes in Dictyostelium. Mol. Cell. Proteomics 14, 66-80. doi: $10.1074 /$ mcp.M114.044560

Shen, L.-N., Lui, H., Dong, C., Xirodimas, D., Naismith, J. H., and Hay, R. T. (2005). Structural basis of NEDD8 ubiquitin discrimination by the deneddylating enzyme NEDP1. EMBO J. 24, 1341-1351. doi: 10.1038/sj.emboj.7600628

Shin, Y.-C., Tang, S.-J., Chen, J.-H., Liao, P.-H., and Chang, S.-C. (2011). The molecular determinants of NEDD8 specific recognition by human SENP8. PLoS One 6:e29899. doi: 10.1371/journal.pone.0029899

Singleton, C. K., Kirsten, J. H., and Dinsmore, C. J. (2006). Function of ammonium transporter A in the initiation of culmination of development in Dictyostelium discoideum. Eukaryot. Cell 5, 991-996. doi: 10.1128/EC.00058-06

Sternfeld, J. (1988). Proportion regulation in Dictyostelium is altered by oxygen. Differentiation 37, 173-179. doi: 10.1111/j.1432-0436.1988.tb00718.x

Sternfeld, J., and Bonner, J. T. (1977). Cell differentiation in Dictyostelium under submerged conditions. Proc. Natl. Acad. Sci. U. S. A. 74, 268-271.

Sternfeld, J., and David, C. N. (1981). Oxygen gradients cause pattern orientation in Dictyostelium cell clumps. J. Cell Sci. 50, 9-17. doi: 10.1242/jcs.50.1.9

Tang, Z., Li, B., Bharadwaj, R., Zhu, H., Ozkan, E., Hakala, K., et al. (2001). APC2 Cullin protein and APC11 RING protein comprise the minimal ubiquitin ligase module of the anaphase-promoting complex. Mol. Biol. Cell 12, 3839-3851. doi: 10.1091/mbc.12.12.3839

Tateishi, K., Omata, M., Tanaka, K., and Chiba, T. (2001). The NEDD8 system is essential for cell cycle progression and morphogenetic pathway in mice. J. Cell Biol. 155, 571-579. doi: 10.1083/jcb.200104035

Tekinay, T., Ennis, H. L., Wu, M. Y., Nelson, M., Kessin, R. H., and Ratner, D. I. (2003). Genetic interactions of the E3 ubiquitin ligase component FbxA with cyclic AMP metabolism and a histidine kinase signaling pathway during Dictyostelium discoideum development. Eukaryot. Cell 2, 618-626. doi: 10.1128/ EC.2.3.618-626.2003

Teng-Umnuay, P., van der Wel, H., and West, C. M. (1999). Identification of a UDP-GlcNAc: Skp1-hydroxyproline GlcNAc-transferase in the cytoplasm of Dictyostelium. J. Biol. Chem. 274, 36392-36402. doi: 10.1074/jbc.274.51.36392

Thomason, P. A., Traynor, D., Cavet, G., Chang, W. T., Harwood, A. J., and Kay, R. R. (1998). An intersection of the cAMP/PKA and two-component signal transduction systems in Dictyostelium. EMBO J. 17, 2838-2845. doi: $10.1093 / \mathrm{emboj} / 17.10 .2838$

van der Wel, H., Morris, H. R., Panico, T., North, S. J., Dell, A., Thomson, J. M., et al. (2001). A non-Golgi $\alpha 1,2$-fucosyltransferase that modifies Skp1 in the cytoplasm of Dictyostelium. J. Biol. Chem. 276, 33952-33963. doi: 10.1074/ jbc.M102555200

Veltman, D. M., Akar, G., Bosgraaf, L., and Van Haastert, P. J. (2009). A new set of small, extrachromosomal expression vectors for Dictyostelium discoideum. Plasmid 61, 110-118. doi: 10.1016/j.plasmid.2008.11.003

Vierstra, R. D. (2012). The expanding universe of ubiquitin and ubiquitin-like modifiers. Plant Physiol. 160, 2-14. doi: 10.1104/pp.112.200667

Vodermaier, H. C. (2004). APC/C and SCF: controlling each other and the cell cycle. Curr. Biol. 14, R787-R796. doi: 10.1016/j.cub.2004.09.020

Wada, H., Kito, K., Caskey, L. S., Yeh, E. T., and Kamitani, T. (1998). Cleavage of the C-terminus of NEDD8 by UCH-L3. Biochem. Biophys. Res. Commun. 251, 688-692. doi: 10.1006/bbrc.1998.9532

Walden, H., Podgorski, M. S., Huang, D. T., Miller, D. W., Howard, R. J., Minor, D. L. Jr., et al. (2003). The structure of the APPBP1-UBA3-NEDD8ATP complex reveals the basis for selective ubiquitin-like protein activation by an E1. Mol. Cell 12, 1427-1437. doi: 10.1016/S1097-2765(03)00452-0

Wang, J., Dubiel, D., Wu, Y., Cheng, Y., Wolf, D. A., and Dubiel, W. (2021). CSN7B defines a variant COP9 signalosome complex with distinct function in DNA damage response. Cell Rep. 34:108662. doi: 10.1016/j.celrep.2020.108662

Wang, B., and Kuspa, A. (2002). CulB, a putative ubiquitin ligase subunit, regulates prestalk cell differentiation and morphogenesis in Dictyostelium spp. Eukaryot. Cell 1, 126-136. doi: 10.1128/EC.1.1.126-136.2002

Wee, S., Geyer, R. K., Toda, T., and Wolf, D. A. (2005). CSN facilitates CullinRING ubiquitin ligase function by counteracting autocatalytic adapter instability. Nat. Cell Biol. 7, 387-391. doi: 10.1038/ncb1241

West, C. M., van der Wel, H., and Wang, Z. A. (2007). Prolyl 4-hydroxylase-1 mediates $\mathrm{O}_{2}$ signaling during development of Dictyostelium. Development 134, 3349-3358. doi: $10.1242 /$ dev.000893

West, C. M., Wang, Z. A., and van der Wel, H. (2010). A cytoplasmic prolyl hydroxylation and glycosylation pathway modifies Skp1 and regulates $\mathrm{O}_{2}$ dependent development in Dictyostelium. Biochim. Biophys. Acta 1800, 160-171. doi: 10.1016/j.bbagen.2009.11.006

Willems, A. R., Schwab, M., and Tyers, M. (2004). A hitchhiker's guide to the cullin ubiquitin ligases: SCF and its kin. Biochim. Biophys. Acta 1695, 133-170. doi: 10.1016/j.bbamcr.2004.09.027

Williams, J. G. (2006). Transcriptional regulation of Dictyostelium pattern formation. EMBO Rep. 7, 694-698. doi: 10.1038/sj.embor.7400714

Wu, J.-T., Lin, H.-C., Hu, Y.-C., and Chien, C.-T. (2005). eddylation and deneddylation regulate Cull and Cul3 protein accumulation. Nat. Cell Biol. 7, 1014-1020. doi: 10.1038/ncb1301

Wu, K., Yamoah, K., Dolios, G., Gan-Erdene, T., Tan, P., Chen, A., et al. (2003). DEN1 is a dual function protease capable of processing the C terminus of Nedd8 and deconjugating hyper-neddylated CUL1. J. Biol. Chem. 278, 28882-28891. doi: 10.1074/jbc.M302888200

Xirodimas, D. P., Sundqvist, A., Nakamura, A., Shen, L., Botting, C., and Hay, R. T. (2008). Ribosomal proteins are targets for the NEDD8 pathway. EMBO Rep. 9, 280-286. doi: 10.1038/embor.2008.10

Xu, X., Pan, M., and Jin, T. (2021). How phagocytes acquired the capability of hunting and removing pathogens from a human body: lessons learned from chemotaxis and phagocytosis of Dictyostelium discoideum (review). Front. Cell Dev. Biol. 9:724940. doi: 10.3389/fcell.2021.817779

Xu, Y., Wang, Z. A., Green, R. S., and West, C. M. (2012). Role of the Skp1 prolyl-hydroxylation/glycosylation pathway in oxygen dependent submerged development of Dictyostelium. BMC Dev. Biol. 12:31. doi: 10.1186/1471-213X-12-31

Yamashita, K., Iriki, H., Kamimura, Y., and Muramoto, T. (2021). CRISPR toolbox for genome editing in Dictyostelium. Front. Cell Dev. Biol. 9:721630. doi: $10.3389 /$ fcell.2021.721630

Ying, J., Zhang, M., Qiu, X., and Lu, Y. (2018). Targeting the neddylation pathway in cells as a potential therapeutic approach for diseases. Cancer Chemother. Pharmacol. 81, 797-808. doi: 10.1007/s00280-018-3541-8

Yu, Z., Kleifeld, O., Lande-Atir, A., Bsoul, M., Kleiman, M., Krutauz, D., et al. (2011). Dual function of Rpn5 in two PCI complexes, the $26 \mathrm{~S}$ proteasome and COP9 signalosome. Mol. Biol. Cell 22, 911-920. doi: 10.1091/mbc E10-08-0655

Zhang, H., He, P., Zhou, Q., Lu, Y., and Lu, B. (2021). The potential oncogenic and MLN4924-resistant effects of CSN5 on cervical cancer cells. Cancer Cell Int. 21:369. doi: 10.1186/s12935-021-02078-5

Zhang, H., Heid, P. J., Wessels, D., Daniels, K. J., Pham, T., Loomis, W. F., et al. (2003). Constitutively active protein kinase A disrupts motility and 
chemotaxis in Dictyostelium discoideum. Eukaryot. Cell 2, 62-75. doi: 10.1128/ EC.2.1.62-75.2003

Zheng, J., Yang, X., Harrell, J. M., Ryzhikov, S., Shim, E. H., Lykke-Andersen, K., et al. (2002). CAND1 binds to unneddylated CUL1 and regulates the formation of SCF ubiquitin E3 ligase complex. Mol. Cell 10, 1519-1526. doi: 10.1016/S1097-2765(02)00784-0

Zimmerman, E. S., Schulman, B. A., and Zheng, N. (2010). Structural assembly of cullin-RING ubiquitin ligase complexes. Curr. Opin. Struct. Biol. 20, 714-721. doi: 10.1016/j.sbi.2010.08.010

Conflict of Interest: The authors declare that the research was conducted in the absence of any commercial or financial relationships that could be construed as a potential conflict of interest.
Publisher's Note: All claims expressed in this article are solely those of the authors and do not necessarily represent those of their affiliated organizations, or those of the publisher, the editors and the reviewers. Any product that may be evaluated in this article, or claim that may be made by its manufacturer, is not guaranteed or endorsed by the publisher.

Copyright $\odot 2022 \mathrm{Kim}$, Mathavarajah and Huber. This is an open-access article distributed under the terms of the Creative Commons Attribution License (CC $B Y)$. The use, distribution or reproduction in other forums is permitted, provided the original author(s) and the copyright owner(s) are credited and that the original publication in this journal is cited, in accordance with accepted academic practice. No use, distribution or reproduction is permitted which does not comply with these terms. 


\section{GLOSSARY}

\begin{tabular}{|c|c|}
\hline AgtA & Alpha-gal-transferase \\
\hline Anapc2 & Anaphase promoting complex subunit 2 \\
\hline CAMP & Cyclic adenosine monophosphate \\
\hline CAND1 & Cullin-associated NEDD8-dissociated protein 1 \\
\hline CAMP & Cyclic adenosine monophosphate \\
\hline Cdk & Cyclin-dependent kinase \\
\hline $\mathrm{CfaD}$ & Counting factor-associated protein D \\
\hline CmfA & Conditioned media factor $\mathrm{A}$ \\
\hline CotC/SP60 & Spore coat protein SP60 \\
\hline CRL & Cullin-RING ligase \\
\hline CSN & COP9 signalosome \\
\hline CUL & Cullin \\
\hline DCUN1D1 & Defective in cullin neddylation 1 \\
\hline EcmA & Extracellular matrix protein $\mathrm{A}$ \\
\hline ErkA & Extracellular signal-regulated kinase \\
\hline Fbx & F-box-containing protein \\
\hline GntA & GlcNAc transferase \\
\hline MAPK & Mitogen-activated protein kinase \\
\hline MTOR & Mammalian target of rapamycin \\
\hline NAE1 & NEDD8 activating enzyme E1 regulatory subunit \\
\hline NEDD8 & Neural precursor cell expressed developmentally downregulated protein 8 \\
\hline PARP1 & Poly(ADP-ribose) polymerase 1 \\
\hline PgtA & Poly-glycosyltransferase \\
\hline PhyA & Prolyl hydroxylase \\
\hline PKA & Protein kinase A \\
\hline RBX1/ROC1 & Ring box $1 /$ regulator of cullins 1 \\
\hline RBX2/ROC2 & Ring box $2 /$ regulator of cullins 2 \\
\hline SCF & Skp1-Cullin-F-box \\
\hline SENP8/DEN1 & Sentrin-specific protease 8 \\
\hline SKP1 & S phase kinase-associated protein 1 \\
\hline SUMO & Small ubiquitin-like modifier \\
\hline UBA3 & Ubiquitin-activating enzyme 3 \\
\hline UBE2F & Ubiquitin-conjugating enzyme E2F \\
\hline UBE2M/UBC12 & Ubiquitin-conjugating enzyme E2M \\
\hline UCHL & Ubiquitin C-terminal hydrolase isozyme \\
\hline UFM1 & Ubiquitin fold modifier 1 \\
\hline URM1 & Ubiquitin-related modifier-1 \\
\hline
\end{tabular}

